\title{
Fault Detection and Isolation for Nonlinear Systems via HOSM Multiple-Observer ${ }^{* \dagger}$
}

\author{
H. Ríos ${ }^{1}$, J. Davila ${ }^{2}$, L. Fridman ${ }^{1 *}$ and C. Edwards ${ }^{3}$ \\ ${ }^{1}$ National Autonomous University of Mexico, Department of Control Engineering and Robotics, Division of Electrical \\ Engineering, Engineering Faculty, C.P. 04510, México, D.F. Mexico. \\ ${ }^{2}$ National Polytechnic Institute, Section of Graduate Studies and Research, ESIME-UPT, C.P. 07340, México, D.F. \\ Mexico. \\ ${ }^{3}$ College of Engineering, Mathematics and Physical Sciences; University of Exeter; North Park Road, Exeter, EX4 4QF, \\ $U K$.
}

\begin{abstract}
SUMMARY
In this paper fault detection and isolation problems are studied for a certain class of nonlinear systems. Under some structural conditions, multiple High-Order Sliding-Mode observers are proposed. The value of the equivalent output injection is used for detecting faults and the multiple-model approach for isolating particular faults in the system. The proposed method provides fast detection and isolation of actuator and plant faults. Simulation results support the proposed approach.
\end{abstract}

KEY WORDS: High-order Sliding-Mode Observers; Fault Detection; Fault Isolation

\section{INTRODUCTION}

\subsection{Antecedents and motivations}

Our society strongly depends on the availability and correct functioning of complex technological processes. Often key processes need to be monitored to prevent improper operation that can damage important components, and, in critical cases, to avoid fatal consequences. The main purpose of fault detection and isolation (FDI) schemes is to indicate that something is wrong (i.e. to generate an alarm when a fault occurs) and then determine which subsystem or component has developed a fault or failure (i.e. identifying its location).

Improvements in modelling techniques have allowed the use of model-based FDI schemes. Such methods have been considered as an effective approach for FDI in theory and in practice (see, for

\footnotetext{
${ }^{*}$ Correspondence to: L. Fridman, National Autonomous University of Mexico, Department of Control Engineering and Robotics, Division of Electrical Engineering, Engineering Faculty, C.P. 04510, México, D.F., Mexico. Email: Ifridman@unam.mx

*A preliminary version of this work has been presented in [1].

${ }^{\dagger}$ The authors gratefully acknowledge the financial support from PAPIIT 17211, CONACyT 56819, 151855 and CVU

270504, FONCICyT 93302, SIP-IPN and CDA-IPN.
} 
example [2], [3] and [4]). In particular, the most commonly employed schemes are observer-based, where the information generated by the observer is used to deduce information about the faults.

In residual generation schemes, the output error between the system and the observer is analyzed to form a residual. When the system is fault free the residual should be small (approximately zero), however when a fault occurs it should become discernably different from zero. An extensive list of publications, describing different FDI methods based on residuals, exists: see for example the survey in [5], fault detection filters in [6], and the tutorial books [2, 3].

Whilst initially work was directed towards linear systems, serious efforts in the last decade have been directed towards nonlinear systems. Observer-based FDI schemes that incorporate different theoretical approaches have been developed. For example the work in [7], [8], [9], and [10], describes differential geometric, algebraic, and feedback control approaches that have been proposed for fault detection. Techniques based on an adaptive estimation approach have also been successfully applied for fault detection, residual generation and fault tolerant control, see for example [11], [12], [13], [14], [15] and [16]. In terms of sliding-mode theory, the most recent contributions have been presented in [17], [18], [19], [20], [21] and [22]. Most of this work formulates the unknown input problem as an actuator or sensor fault reconstruction problem. In particular, High-Order-Sliding-Mode (HOSM) techniques are applied in [23] to decrease the level of uncertainty in a Linear-Parameter-Varying system, which leads to the improvement of setmembership estimates generated by an interval observer. This approach is applied to fault detection by verifying the consistency between the output trajectory and its estimated domain. In [24], a FDI scheme based on HOSM techniques for a three tanks system is proposed. The HOSM observer design allows the estimation of the magnitude of a relative degree one actuator faults. A residual generation approach, using the estimated parameters, inputs, outputs and their estimated derivatives obtained by means of the HOSM differentiator [25], is presented in [26]. All the approaches in the three papers described above are restricted to only one type of fault with a fixed relative degree. In the work described in this paper, we exploit the qualities associated with the HOSM methods given by [25], and add a multi-model approach that allows us to isolate faults even if the relative degree vector changes as a consequence of the faults.

The multiple-model based approach has also attracted much attention in the last decade. There are many advantages of using a multiple-model approach for fault diagnosis in complex systems (see, for example [27], [28] and [29]). The main reason is because it is often impractical to represent all possible system failure behaviour by a single model. Moreover, since the number of failure modes is finite, it is possible to employ a control algorithm that has been specifically designed for each fault scenario. Of course some applications may require a large number of modes to represent all possible faults, which results in high computational costs. Nevertheless, the multiple-model approach has been applied to many FDI problems in aerospace systems, including the aircraft flight control systems [30, 31] and inertial navigation systems [32].

The main idea of the multiple-model approach for FDI is as follows: a model set must be created that contains all the different plausible fault conditions in the system in the form of local models or sub-models. In addition, the model set usually includes the nominal fault free model. Faults are identified by estimating which of the possible local model is most valid using multiple-model estimation algorithms. When there are no faults present in the system, the nominal model will be 
valid. In case of an a-priori considered fault, one of the other models in the model set will become valid.

\subsection{Main Contribution}

Motivated by the above, and taking into account that multiple-model based FDI schemes have already been successfully used in many applications, the main contribution of this paper is:

- A FDI approach that allows us to estimate the system state in finite time, and solve the FDI problem, even if the relative degree vector of the output w.r.t. the fault changes among the possible faulty scenarios.

For fulfilling the above mentioned goal the following methodology has been proposed in this manuscript:

1. A HOSM Multiple-Observer for estimating the state of a class of nonlinear systems in finite time.

2. Exploit the equivalent output injection of the HOSM observers to solve the fault detection problem.

3. Use the multiple-model approach to solve the fault isolation problem for such systems.

This means that a new methodology which consists in a combination between HOSM MultipleObserver and multiple-model approach is proposed. Such methodology exploits the qualities associated with the HOSM methods to detect faults, and the multi-model approach allows us to isolate them even if the relative degree vector changes as a consequence of the faults. With respect to the already existing methods, most of the approaches are restricted to only one type of fault with a fixed relative degree.

\subsection{Structure of the paper}

The paper has the following structure. Section 2 deals with the problem statement. In Section 3 the preliminaries are presented. Section 4 describes the design of the HOSM Multiple-Observer and the main assumptions of the proposed approach, whilst in Section 5 the FDI scheme is presented. A simulation example is given in Section 6 and finally, some concluding remarks are given in Section 7.

\subsection{Notation}

The set $\Re^{+}$is defined as $\Re^{+}=\{\varsigma \in \Re: \varsigma \geq 0\}$. Denote by $\mathcal{L}_{\infty}$ the set of all inputs $\zeta$ that satisfy $\|\zeta\|<\infty$. With reference to a scalar function $h$ with a vector argument $x$ defined in an open set $\Omega \in \Re^{n}$ such that $h(x): \Re^{n} \rightarrow \Re$, define $d h(x)=\frac{\partial h(x)}{\partial x}=\left[\begin{array}{lll}\frac{\partial h(x)}{\partial x_{1}} & \cdots & \frac{\partial h(x)}{\partial x_{n}}\end{array}\right]$. Also define $L_{f(x)} h(x)=d h(x) f(x)$ as the Lie derivative of $h(x)$ along $f(x)$, and the $k-t h$ Lie derivative as $L_{f(x)}^{k} h(x)=d\left(L_{f(x)}^{k-1} h(x)\right) f(x)$. Finally, the function $\lceil\xi\rfloor^{r}$ is defined as $\lceil\xi\rfloor^{r}=|\xi|^{r} \operatorname{sign}(\xi)$. 


\section{PROBLEM STATEMENT}

Consider the following nonlinear system subject to faults

$$
\begin{aligned}
& \dot{x}(t)=f(x(t))+B u(t)+F_{\kappa}(x(t)) \omega_{\kappa}(t), \quad \kappa=1, \ldots, q, \\
& y(t)=h(x(t)), \psi(t)=y(t)+v(t),
\end{aligned}
$$

where $x(t) \in \mathcal{X} \subseteq \Re^{n}$ is the state vector, $u(t) \in \mathcal{U} \subseteq \Re^{m}$ is the control input vector, $y(t) \in \mathcal{Y} \subseteq \Re^{p}$ is the output, and $v(t) \in \Re^{p}$ is a Lebesgue-measurable sampling noise. The quantity $\psi(t)$ represents the available signal obtained from real-time measurements. The vector field $f(x(t))$ and the function $h(x(t))=\left[h_{1}(x(t)) \cdots h_{p}(x(t))\right]^{T}$ are assumed to be sufficiently smooth and the matrix $B=\left[B_{1} \cdots B_{m}\right] \in \Re^{n \times m}$ is a known constant matrix.

The fault scenarios are considered to be known a-priori and it is assumed that their effects can be modelled as additive terms of the form:

$$
F_{\kappa}(x(t)) \omega_{\kappa}(t) \in \mathcal{F}, \text { where } \mathcal{F}=\left\{F_{1}(x(t)) \omega_{1}(t), \ldots, F_{q}(x(t)) \omega_{q}(t)\right\}
$$

i.e. there exist $q$ different possible fault cases represented by $q$ faulty components $F_{\kappa}(x(t)) \omega_{\kappa}(t)$ that can change the system properties (plant faults) and/or the dynamical input properties of the system (actuator faults). Assume that $\omega_{\kappa}(t) \in \Re^{p}$, and that the $p$ vector fields of $F_{\kappa}(x(t))$, i.e. $F_{\kappa}(x(t))=\left[F_{\kappa, 1}(x(t)), \ldots, F_{\kappa, p}(x(t))\right]$, are smooth almost everywhere. It is assumed that all the faults under consideration allow the existence and uniqueness of solutions to the whole semi-axis $t \geq 0$

The aim of this paper is to detect and isolate the presence of faults in system (1). To achieve this aim, a bank of HOSM observers is proposed.

\section{PRELIMINARIES}

Consider system (1) in the fault-free case, i.e. when $F_{\kappa}(x(t)) \omega_{\kappa}(t)=0$. The following relative degree definition is introduced [33]:

\section{Definition 1}

The system output $y(t)$ has a (vector) relative degree $\left(r_{1}, \ldots, r_{p}\right)$ at a point $x^{0}$, if

$$
\begin{aligned}
& d\left(L_{f(x)}^{k} h_{i}(x)\right) B_{j}=0, \quad \forall j=1, \ldots, m, \forall k<r_{i}-1, \forall i=1, \ldots, p, \\
& d\left(L_{f(x)}^{r_{i}-1} h_{i}(x)\right) B_{j} \neq 0, \text { for at least one } 1 \leq j \leq m, \forall i=1, \ldots, p .
\end{aligned}
$$

for all $x$ in a neighborhood of $x^{0}$.

\subsection{HOSM Differentiator}

The results presented in this section have to be understood in a component-wise sense. In order to estimate the derivatives of a signal $y(t)$ based on the measurements $\psi(t)=y(t)+v(t)$, where $v(t)$ 
is a bounded Lebesgue measurable signal, the following HOSM differentiator [25] is introduced:

$$
\begin{aligned}
& \dot{\vartheta}_{1}=\vartheta_{2}-\alpha_{1} M^{\frac{1}{r}}\left\lceil\vartheta_{1}-\psi(t)\right\rfloor^{\frac{r-1}{r}}, \\
& \dot{\vartheta}_{i}=\vartheta_{i+1}-\alpha_{i} M^{\frac{1}{r-i+1}}\left\lceil\vartheta_{i}-\dot{\vartheta}_{i-1}\right\rfloor^{\frac{r-i}{r-i+1}}, i=2, \ldots, r-1, \\
& \dot{\vartheta}_{r}=-\alpha_{r} M\left\lceil\vartheta_{r}-\dot{\vartheta}_{r-1}\right\rfloor^{0},
\end{aligned}
$$

where $M$ is an upper bound of $y^{(r)}(t)$, and the set of gains $\left\{\alpha_{k}\right\}_{k=1}^{r}$ are chosen recursively and sufficiently large. In particular, according to [25], one possible choice is $\alpha_{6}=1.1, \alpha_{5}=1.5, \alpha_{4}=2$, $\alpha_{3}=3, \alpha_{2}=5, \alpha_{1}=8$ (for the case when $r \leq 6$ ). The following theorem is taken from [34], and describes the properties of the HOSM differentiator in the presence of noise and sample time, is introduced.

\section{Theorem 1}

Consider the HOSM differentiator (5) of order $r-1$, with proper parameters $\alpha_{k}$. Let

$$
\left|y^{(r)}(t)\right|<M,|v(t)| \leq \kappa_{v} M \xi^{r},
$$

where $\xi$ is a positive parameter. Suppose that $\psi$ is sampled with a possibly variable time step $\tau_{s}>0$, and $\tau_{s} \leq \kappa_{\tau} \xi$, with $\kappa_{v}, \kappa_{\tau}$ being some positive constants. Then for any positive constants $\gamma_{1}, \gamma_{2}, \ldots, \gamma_{r}$ and any $\kappa_{\psi}, 0<\kappa_{\psi}<\gamma_{1}$, there exist $\kappa_{v}, \kappa_{\tau}, \gamma_{t}>0$, such that if the inequality

$$
\left|\vartheta_{1}-\psi(t)\right| \leq \kappa_{\psi} M \xi^{r},
$$

holds at all sampling instants within the finite-time interval of the length $\gamma_{t} \xi$, then starting from the beginning of this interval the inequalities

$$
\left|\vartheta_{k}-y^{(k-1)}(t)\right| \leq \gamma_{k} M \xi^{r-k+1}, \quad k=1, \ldots, r,
$$

hold and are kept forever.

\section{Remark 1}

Note that with discrete sampling the differential equations are replaced by their Euler approximations. Furthermore the differentiator (5) provides the best possible asymptotic accuracy in the presence of input noises [25]. Reliable derivatives/estimates are only available after a finitetime transient $\gamma_{t} \xi$.

The HOSM differentiator given in (5) provides the derivatives up to order $r-1$ by means of the vector $\vartheta$, i.e. $\left[\vartheta_{1} \vartheta_{2} \ldots \vartheta_{r}\right]^{T}=\left[\hat{y}(t) \dot{\hat{y}}(t) \ldots \hat{y}^{(r-1)}(t)\right]^{T}$, where $\left\{\hat{y}^{(k)}\right\}_{k=0}^{r-1}$ are the estimated derivative signals. Notice that in the absence of noise and sample time, the HOSM differentiator (5) ensures the exact estimation of the derivatives in finite-time.

\section{HOSM MULTIPLE-OBSERVER DESIGN}

In this section different fault cases which can appear in the system (1) are studied. Then, the conditions required to design the bank of HOSM observers are established for each case. 


\subsection{Fault-Free Case}

First consider the fault-free case. Then, system (1) takes the following structure

$$
\begin{aligned}
& \dot{x}(t)=f(x(t))+B u(t), \\
& y(t)=h(x(t)), \psi(t)=y(t)+v(t) .
\end{aligned}
$$

Define the mapping $\Phi(x(t))$ and its corresponding Jacobian matrix as follows

$$
\Phi(x(t))=\left[\begin{array}{c}
h_{1}(x(t)) \\
L_{f(x(t))} h_{1}(x(t)) \\
\vdots \\
L_{f(x(t))}^{r_{1}-1} h_{1}(x(t)) \\
\vdots \\
h_{p}(x(t)) \\
L_{f(x(t))} h_{p}(x(t)) \\
\vdots \\
L_{f(x(t))}^{r_{p}-1} h_{p}(x(t))
\end{array}\right] \Rightarrow \frac{\partial \Phi(x(t))}{\partial x(t)}=\left[\begin{array}{c}
d h_{1}(x(t)) \\
d L_{f(x(t))} h_{1}(x(t)) \\
\vdots \\
d L_{f(x(t))}^{r_{1}-1} h_{1}(x(t)) \\
\vdots \\
d h_{p}(x(t)) \\
d L_{f(x(t))} h_{p}(x(t)) \\
\vdots \\
d L_{f(x(t))}^{r_{p}-1} h_{p}(x(t))
\end{array}\right] .
$$

\section{Assumption 1}

Assume that relative degree condition (4) is satisfied by system (9), and the Jacobian matrix in (10) is such that $\operatorname{rank}\left(\frac{\partial \Phi(x(t))}{\partial x(t)}\right)=n, \forall x \in \mathcal{X}$.

\section{Remark 2}

The fulfilment of Assumption 1 implies local observability of system (1), i.e. it is observable $\forall x \in \mathcal{X}$. Notice that if Assumption 1 is satisfied, then the mapping $\Phi(x)$ is a local diffeomorphism on $\mathcal{X}$. Moreover, if $\mathcal{X}=\Re^{n}$, then $\Phi(x(t))$ is a global diffeomorphism (see for example [33]), and then the system in (1) is observable $\forall x \in \Re^{n}$.

Suppose that Assumption 1 is satisfied. Then, it is possible to design the following observer

$$
\begin{aligned}
& \dot{\bar{x}}(t)=f(\bar{x}(t))+B u(t)+\left(\frac{\partial \Phi(\bar{x}(t))}{\partial \bar{x}(t)}\right)^{-1} \nu, \\
& \bar{y}(t)=h(\bar{x}(t)),
\end{aligned}
$$

with the estimated state vector $\bar{x}(t) \in \Re^{n}$, the estimated output $\bar{y}(t) \in \Re^{p}$, and the correction term $\nu \in \Re^{n}$ that will be described in the sequel. The solutions of (11) are understood in the Filippov sense [35], to provide the possibility of using discontinuous signals in the $\nu$ term. These solutions coincide with the usual solutions, when the right-hand sides are continuous. It is also assumed that all the considered correction terms allow the existence and extension of solutions to the whole semiaxis $t \geq 0$.

Let the following assumption be satisfied: 


\section{Assumption 2}

There is a set of known constants $\left\{M_{i}\right\}_{i=1}^{p}>0$ such that the following inequalities are satisfied:

$$
\left|\left\{L_{f(\bar{x})}^{r_{i}} h_{i}(\bar{x})-L_{f(x)}^{r_{i}} h_{i}(x)\right\}_{i=1}^{p}\right|<\left\{M_{i}\right\}_{i=1}^{p} .
$$

\section{Remark 3}

From a physical point of view (for example, in mechanical systems), Assumption 2 implies the knowledge of an upper bound on the acceleration and a bound for the initial estimation error. This is not particularly restrictive since most physical signals are bounded and usually it is possible to estimate a-priori with a reasonable level of accuracy the initial conditions of the system trajectories. Notice that Assumption 2 is satisfied if the system (1) is Bounded-Input Bounded-State (BIBS); see for example [36].

The HOSM differentiator in (5) is used as auxiliary dynamics. The differentiator-based auxiliary dynamics takes the following form for each output

$$
\begin{aligned}
& \dot{\vartheta}_{1,1}=\vartheta_{1,2}-\alpha_{1,1} M_{1}^{\frac{1}{r_{1}}}\left\lceil e_{y_{1}}(t)\right\rfloor^{\frac{r_{1}-1}{r_{1}}}, \\
& \dot{\vartheta}_{1, j}=\vartheta_{1, j+1}-\alpha_{1, j} M_{1}^{\frac{1}{r_{1}-i+1}}\left\lceil\vartheta_{1, j}-\dot{\vartheta}_{1, j-1}\right\rfloor^{\frac{r_{1}-i}{r_{1}-i+1}}, i=2, \ldots, r_{1}-1, \\
& \dot{\vartheta}_{1, r_{1}}=-\alpha_{1, r_{1}} M_{1}\left\lceil\vartheta_{1, r_{1}}-\dot{\vartheta}_{1, r_{1}-1}\right\rfloor^{0}, \\
& \quad \vdots \\
& \dot{\vartheta}_{p, 1}=\vartheta_{p, 2}-\alpha_{p, 1} M_{p}^{\frac{1}{r_{p}}}\left\lceil e_{y_{p}}(t)\right\rfloor^{\frac{r_{p}-1}{r_{p}}}, \\
& \dot{\vartheta}_{p, j}=\vartheta_{p, j+1}-\alpha_{p, j} M_{p}^{\frac{1}{r_{p}-i+1}}\left\lceil\vartheta_{p, j}-\dot{\vartheta}_{p, j-1}\right\rfloor^{\frac{r_{p}-i}{r_{p}-i+1}}, i=2, \ldots, r_{p}-1, \\
& \dot{\vartheta}_{p, r_{p}}=-\alpha_{p, r_{p}} M_{p}\left\lceil\vartheta_{p, r_{p}}-\dot{\vartheta}_{p, r_{p}-1}\right\rfloor^{0},
\end{aligned}
$$

where $\left\{e_{y_{i}}(t)\right\}_{i=1}^{p}=\left\{\bar{y}_{i}(t)-\psi_{i}(t)\right\}_{i=1}^{p}$, are the output errors. Then, the correction term is taken from (13) as:

$$
\nu=\left[\begin{array}{c}
-\alpha_{1,1} M_{1}^{\frac{1}{r_{1}}}\left\lceil e_{y_{1}}(t)\right\rfloor^{\frac{r_{1}-1}{r_{1}}} \\
-\alpha_{1, j} M_{1}^{\frac{1}{r_{1}-i+1}}\left\lceil\vartheta_{1, j}-\dot{\vartheta}_{1, j-1}\right\rfloor^{\frac{r_{1}-i}{r_{1}-i+1}}, i=2, \ldots, r_{1}-1 \\
-\alpha_{1, r_{1}} M_{1}\left\lceil\vartheta_{1, r_{1}}-\dot{\vartheta}_{1, r_{1}-1}\right\rfloor^{0} \\
\vdots \\
-\alpha_{p, 1} M_{p}^{\frac{1}{r_{p}}}\left\lceil e_{y_{p}}(t)\right\rfloor^{\frac{r_{p}-1}{r_{p}}} \\
-\alpha_{p, j} M_{p}^{\frac{1}{r_{p}-i+1}}\left\lceil\vartheta_{p, j}-\dot{\vartheta}_{p, j-1}\right\rfloor^{\frac{r_{p}-i}{r_{p}-i+1}}, i=2, \ldots, r_{p}-1 \\
-\alpha_{p, r_{p}} M_{p}\left\lceil\vartheta_{p, r_{p}}-\dot{\vartheta}_{p, r_{p}-1}\right\rfloor^{0}
\end{array}\right] .
$$

The constants $\left\{M_{i}\right\}_{i=1}^{p}$ are chosen such that Assumption 2 is satisfied, and $\left\{\alpha_{i, j}\right\}_{i=1, j=1}^{p, r_{i}}$ are chosen as in (5). Notice that the HOSM-based controllers are powerful tools when used in observation schemes, given that the chattering effects are reduced to 'computational chattering' which is harmless to the system.

Taking into account the previous explanations, the following lemma can be stated: 


\section{Lemma 1}

Let the observer in (11) with the correction term in (14) be applied to system (9) and suppose Assumption 1 is satisfied. Then, provided the gains $\left\{M_{i}\right\}_{i=1}^{p}$ are selected such that Assumption 2 holds; and the parameters $\left\{\alpha_{i, k}\right\}_{i=1, k=1}^{p, r_{i}}$ are chosen according to [25], the state estimation error converges to a $\beta$-neighborhood of the origin, dependent on the amplitude of the noise, in finite-time, i.e. $\|x(t)-\bar{x}(t)\| \leq \beta$, for $\beta>0$ and $\forall t>t^{* \dagger}$.

Proof

Denote $\left\{\bar{y}_{i}^{(j-1)}(t)-y_{i}{ }^{(j-1)}(t)\right\}_{i=1, j=1}^{p, r_{i}}=\left\{M_{i} \epsilon_{i, j}(t)\right\}_{i=1, j=1}^{p, r_{i}}$. Then, due to the auxiliary dynamics (13), the output observation error dynamics takes the following form:

$$
\begin{aligned}
& M_{1} \dot{\epsilon}_{1,1}=M_{1} \epsilon_{1,2}-\alpha_{1,1} M_{1}^{\frac{1}{r_{1}}}\left\lceil M_{1} \epsilon_{1,1}-v_{1}(t)\right\rfloor^{\frac{r_{1}-1}{r_{1}}}, \\
& M_{1} \dot{\epsilon}_{1, j}=M_{1} \epsilon_{1, j+1}-\alpha_{1, j} M_{1}^{\frac{1}{r_{1}-j+1}}\left\lceil M_{1} \epsilon_{1, j}-M_{1} \dot{\epsilon}_{1, j-1}\right\rfloor^{\frac{r_{1}-j}{r_{1}-j+1}}, j=2, \ldots, r_{1}-1, \\
& M_{1} \dot{\epsilon}_{1, r_{1}}=\Psi_{1}(\bar{x}, x, u)-\alpha_{1, r_{1}} M_{1}\left\lceil M_{1} \epsilon_{1, r_{1}}-M_{1} \dot{\epsilon}_{1, r_{1}-1}\right\rfloor^{0}, \\
& \quad \vdots \\
& M_{p} \dot{\epsilon}_{p, 1}=M_{p} \epsilon_{p, 2}-\alpha_{p, 1} M_{p}^{\frac{1}{r_{p}}}\left\lceil M_{p} \epsilon_{p, 1}-v_{p}(t)\right\rfloor^{\frac{r_{p}-1}{r_{p}}}, \\
& M_{p} \dot{\epsilon}_{p, j}=M_{p} \epsilon_{p, j+1}-\alpha_{p, j} M_{p}^{\frac{1}{r_{p}-j+1}}\left\lceil M_{p} \epsilon_{p, j}-M_{p} \dot{\epsilon}_{p, j-1}\right\rfloor^{\frac{r_{p}-j}{r_{p}-j+1}}, j=2, \ldots, r_{p}-1, \\
& M_{p} \dot{\epsilon}_{p, r_{p}}=\Psi_{p}(\bar{x}, x, u)-\alpha_{p, r_{p}} M_{p}\left\lceil M_{p} \epsilon_{p, r_{p}}-M_{p} \dot{\epsilon}_{p, r_{p}-1}\right\rfloor^{0},
\end{aligned}
$$

where $\left\{\Psi_{i}(\bar{x}, x, u)\right\}_{i=1}^{p}=\left\{L_{f(\bar{x})}^{r_{i}} h_{i}(\bar{x})-L_{f(x)}^{r_{i}} h_{i}(x)\right\}_{i=1}^{p}$. Dividing by $M_{i}$ (15), the following differential inclusion is obtained

$$
\begin{aligned}
& \dot{\epsilon}_{1,1}=\epsilon_{1,2}-\alpha_{1,1}\left\lceil\epsilon_{1,1}-v_{1}(t) / M_{1}\right\rfloor^{\frac{r_{1}-1}{r_{1}}}, \\
& \dot{\epsilon}_{1, j}=\epsilon_{1, j+1}-\alpha_{1, j}\left\lceil\epsilon_{1, j}-\dot{\epsilon}_{1, j-1}\right\rfloor^{\frac{r_{1}-j}{r_{1}-j+1}}, j=2, \ldots, r_{1}-1, \\
& \dot{\epsilon}_{1, r_{1}} \in[-1,1]-\alpha_{1, r_{1}}\left\lceil\epsilon_{1, r_{1}}-\dot{\epsilon}_{1, r_{1}-1}\right\rfloor^{0}, \\
& \quad \vdots \\
& \dot{\epsilon}_{p, 1}=\epsilon_{p, 2}-\alpha_{p, 1}\left\lceil\epsilon_{p, 1}-v_{p}(t) / M_{p}\right\rfloor^{\frac{r_{p}-1}{r_{p}}}, \\
& \dot{\epsilon}_{p, j}=\epsilon_{p, j+1}-\alpha_{p, j}\left\lceil\epsilon_{p, j}-\dot{\epsilon}_{p, j-1}\right\rfloor^{\frac{r_{p}-j}{r_{p}-j+1}}, j=2, \ldots, r_{p}-1, \\
& \dot{\epsilon}_{p, r_{p}} \in[-1,1]-\alpha_{p, r_{p}}\left\lceil\epsilon_{p, r_{p}}-\dot{\epsilon}_{p, r_{p}-1}\right\rfloor^{0},
\end{aligned}
$$

where the last row of each inclusion block follows from Assumption 2, i.e. $\left\{\Psi_{i}(\bar{x}, x, u)\right\}_{i=1}^{p} \in$ $\left\{\left[-M_{i}, M_{i}\right]\right\}_{i=1}^{p}$. Therefore, from Theorem 1, there exist parameters $\left\{\alpha_{i, j}\right\}_{i=1, j=1}^{p, r_{i}}$ and $t^{*}>0$ such that for all $t \geq t^{*}$

$$
\left|\left\{\epsilon_{i, j}(t)\right\}_{i=1, j=1}^{p, r_{i}}\right| \leq\left\{\gamma_{i, j} M_{i} \xi^{r_{i}-j+1}\right\}_{i=1, j=1}^{p, r_{i}},
$$

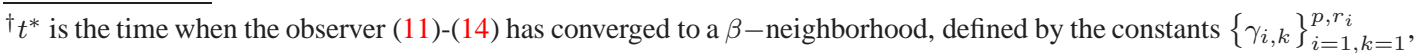
$M_{i}$, and the amplitude of the noise. 
for some positive constants $\left\{\gamma_{i, k}\right\}_{i=1, k=1}^{p, r_{i}}$. Then, according to [25], each element of the dynamics in (16) converges to a region close to zero that is proportional to a certain order of the noise, in finite-time ${ }^{\ddagger}$. Notice that each $\epsilon_{i, k}(t)$ is bounded by $\gamma_{i, k} M_{i} \xi^{r_{i}-k+1}$, in other words, $\epsilon_{i, k}(t)$ is inside a neighborhood of radius $\gamma_{i, k} M_{i} \xi^{r_{i}-k+1}$. Then, according to Assumption 1, it is possible to find a $\beta$-neighborhood of $x$, i.e.

$$
N(x, \beta)=\left\{\bar{x}(t) \in \Re^{n} \mid\|x(t)-\bar{x}(t)\| \leq \beta\right\} .
$$

It is clear that the value of $\beta$ depends on the constants $\left\{\gamma_{i, k}\right\}_{i=1, k=1}^{p, r_{i}}$ and on the way in which the elements of $\nu$ are combined due to the matrix $\left(\frac{\partial \Phi(\bar{x}(t))}{\partial \bar{x}(t)}\right)^{-1}$. Nevertheless, $\beta$ can always be calculated since it depends on the parameters of the HOSM differentiator and on the amplitude of the noise.

In order to know when the observer converges there exist some analytical reaching time estimations (see, for example [37] and [38]), for the case $r_{i}=2$, i.e. for the Super-Twisting algorithm [39]. Nevertheless, such estimations are very crude, so that in practice it is better to obtain its value in the following way.

According to [34], to detect when the observer converges to the $\beta$-neighborhood, proportional to certain order of the noise, it is sufficient to verify that the following inequalities are satisfied

$$
\left|e_{y_{i}}(t)\right| \leq \gamma_{i, 1} M_{i} \xi^{r_{i}}, \quad \forall t \in\left[0, \gamma_{t_{i}} \xi\right], \quad \forall i=1, \ldots, p
$$

It is usual to estimate the constants $\gamma_{i, 1}$ and $\gamma_{t}$ through simulation. Therefore, when all the output errors $e_{y_{i}}(t)$ are inside its respective error band $\gamma_{i, 1} M_{i} \xi^{r_{i}}$ during the time interval $\left[0, \gamma_{t_{i}} \xi\right]$, the observer will have converged, and $t^{*}$ will be the time at which all these inequalities are satisfied, i.e.

$$
t^{*}=\max _{\forall i=1, \ldots, p} \gamma_{t_{i}} \xi
$$

\subsection{Plant Fault Case}

Consider the case when system (1) has a plant fault, i.e. $F_{\kappa}(x(t)) \omega_{\kappa}(t)=\Delta_{\kappa} f_{\kappa}(x(t))$, with $\Delta_{\kappa} \in$ $\Re^{n \times n}$ known distribution matrices and $f_{\kappa}(x(t))$ known faulty vector fields. Then, system (1) takes the following structure

$$
\begin{aligned}
& \dot{x}(t)=f_{\Delta, \kappa}(x(t))+B u(t), \\
& y(t)=h(x(t)), \psi(t)=y(t)+v(t),
\end{aligned}
$$

\footnotetext{
$¥$ Due to the discontinuous nature of the proposed HOSM observer, the solution of the differential inclusion (16) is understood in the Filippov sense [35]. These solutions coincide with the usual solutions, when the right-hand sides are continuous. For the particular employed HOSM algorithm, the convergence of a unique solution is given by [25] based on homogeneity properties.
} 
where $f_{\Delta, \kappa}(x(t))=f(x(t))+\Delta_{\kappa} f_{\kappa}(x(t))$. Let Assumption 1 be satisfied with the new vector field $f_{\Delta, \kappa}(x(t))$. Then, it is possible to design an observer of the form

$$
\begin{aligned}
& \dot{\bar{x}}(t)=f_{\Delta, \kappa}(\bar{x}(t))+B u(t)+\left(\frac{\partial \Phi(\bar{x}(t))}{\partial \bar{x}(t)}\right)^{-1} \nu, \\
& \bar{y}(t)=h(\bar{x}(t))
\end{aligned}
$$

where the correction term $\nu$ is designed according to (14) with a new set of gains $\left\{M_{i, f_{\Delta, \kappa}}\right\}_{i=1}^{p}$ satisfying the following assumption.

\section{Assumption 3}

There is a set of known constants $\left\{M_{i, f_{\Delta, \kappa}}\right\}_{i=1}^{p}>0$ such that the following inequalities are satisfied:

$$
\left|\left\{L_{f_{\Delta, \kappa}(\bar{x})}^{r_{i}} h_{i}(\bar{x})-L_{f_{\Delta, \kappa}(x)}^{r_{i}} h_{i}(x)\right\}_{i=1}^{p}\right|<\left\{M_{i, f_{\Delta, \kappa}}\right\}_{i=1}^{p} .
$$

Then, according to Lemma 1 , the state estimation error converges to a $\beta_{f_{\Delta, \kappa}}$-neighborhood, proportional to a certain order of the noise, in finite-time, i.e. $\|x(t)-\bar{x}(t)\| \leq \beta_{f_{\Delta, \kappa}}$, for $\beta_{f_{\Delta, \kappa}}>0$ and $\forall t>t_{f_{\Delta, \kappa}}^{*}$.

\subsection{Actuator Fault Case}

Consider the case when system (1) has an actuator fault, i.e. $F_{\kappa}(x(t)) \omega_{\kappa}(t)=B_{a_{\kappa}} u(t)$, where the $B_{a_{\kappa}}$ are known matrices. Then, system (1) takes the following structure

$$
\begin{aligned}
& \dot{x}(t)=f(x(t))+B_{\Delta, \kappa} u(t), \\
& y(t)=h(x(t)), \psi(t)=y(t)+v(t),
\end{aligned}
$$

where $B_{\Delta, \kappa}=B+B_{a_{\kappa}}$. Let Assumption 1 be satisfied for the new input matrix $B_{\Delta, \kappa}$. Then, it is possible to design the observer

$$
\begin{aligned}
& \dot{\bar{x}}(t)=f(\bar{x}(t))+B_{\Delta, \kappa} u(t)+\left(\frac{\partial \Phi(\bar{x}(t))}{\partial \bar{x}(t)}\right)^{-1} \nu, \\
& \bar{y}(t)=h(\bar{x}(t)),
\end{aligned}
$$

where the correction term $\nu$ is designed according to (14) with a new set of gains $\left\{M_{i, B_{\Delta, \kappa}}\right\}_{i=1}^{p}$ satisfying the following assumption:

\section{Assumption 4}

There are known constants $\left\{M_{i, B_{\Delta, \kappa}}\right\}_{i=1}^{p}>0$ such that the following inequalities are satisfied:

$$
\left|\left\{L_{f(\bar{x})}^{r_{i}} h_{i}(\bar{x})-L_{f(x)}^{r_{i}} h_{i}(x)\right\}_{i=1}^{p}\right|<\left\{M_{i, B_{\Delta, \kappa}}\right\}_{i=1}^{p} .
$$

\footnotetext{
$\S_{t_{\Delta, \kappa}^{*}}^{*}$ is the time when the observer (22)-(14) has converged to a $\beta_{f_{\Delta, \kappa}}$-neighborhood, defined by the constants $\left\{\gamma_{i, j}\right\}_{i=1, j=1}^{p, r_{i}},\left\{M_{i, f_{\Delta, \kappa}}\right\}_{i=1}^{p}$, and amplitude of the noise. Notice that $t_{f_{\Delta, \kappa}}^{*}$ can be calculated by (20).
} 
Therefore, according to Lemma 1 , the state estimation error converges to a $\beta_{B_{\Delta, \kappa}}$-neighborhood, proportional to certain order of the noise, in finite-time, i.e. $\|x(t)-\bar{x}(t)\| \leq \beta_{B_{\Delta, \kappa}}$, for $\beta_{B_{\Delta, \kappa}}>0$ and $\forall t>t_{B_{\Delta, \kappa}}^{*}$.

Arguing as in Sections 4.1-4.3, it is possible to design the following bank of $q+1$ HOSM observers:

$$
\begin{aligned}
& \dot{\bar{x}}_{\lambda}(t)=f_{\lambda}\left(\bar{x}_{\lambda}(t)\right)+B_{\lambda} u(t)+\left(\frac{\partial \Phi_{\lambda}\left(\bar{x}_{\lambda}(t)\right)}{\partial \bar{x}_{\lambda}(t)}\right)^{-1} \nu_{\lambda}, \\
& \bar{y}_{\lambda}(t)=h\left(\bar{x}_{\lambda}(t)\right),
\end{aligned}
$$

with estimated state vectors $\bar{x}_{\lambda}(t) \in \Re^{n}$, estimated outputs $\bar{y}_{\lambda}(t) \in \Re^{p}, \lambda \in \Lambda=\{0,1, \ldots, q\}$, and the correction terms $\nu_{\lambda} \in \Re^{n}$. The structure for $f_{\lambda}$ and $B_{\lambda}$ depends on the kind of faults considered ${ }^{\|}$, as will be seen later in the simulation example.

\section{Remark 4}

Notice that to design the observers it is only necessary to calculate the inverse matrices $\left(\frac{\partial \Phi(\bar{x})}{\partial \bar{x}}\right)^{-1}$, not the inverse transformations $\Phi^{-1}(\bar{z})$.

\section{Remark 5}

It is important to note that the vector relative degree can change due to the different faults. However, while Assumption 1 is satisfied, it will always be possible to design an observer that converges in finite-time.

\section{Remark 6}

Notice that in the absence of noise and sampling, observers (27) ensures the exact estimation of the corresponding state in finite-time.

Notice that if the observability mappings defined in (10) are equal for every $\lambda$, it is possible to design only one observer (selecting correctly the gains $M_{\lambda_{i}}$ of the correction term**) that will be capable of estimating the state of the system, with an error proportional to certain order of the noise, independently of the faults that occur. However, this only solves the problem of state estimation, not the problem of FDI that is the topic of this paper.

On the other hand, when a bank of observers is designed, irrespective of whether the observability mappings are equal or different, it is possible that more than one of the $q+1$ observers estimates the state. Nevertheless, the equivalent output injection will be different for each of them; this is the key point in order to solve the FDI problem, and will be discussed in the sequel.

\footnotetext{
ฯ $t_{B_{\Delta, \kappa}}^{*}$ is the time when the observer (25)-(14) has converged to a $\beta_{B_{\Delta, \kappa}}$-neighborhood, defined by the constants $\left\{\gamma_{i, k}\right\}_{i=1, k=1}^{p, r_{i}},\left\{M_{i, B_{\Delta, \kappa}}\right\}_{i=1}^{p}$, and amplitude of the noise. Notice that $t_{B_{\Delta, \kappa}}^{*}$ can be calculated by (20).

$\|$ The observer $\lambda=0$ is for the fault-free case.

** For this purpose, it is necessary to check the corresponding assumption of boundedness for every $\lambda$ (Assumption 2, 3 or 4 , depending on the scenario), then, the maximum value of $M_{i}$ is selected such that satisfies all assumptions of boundedness.
} 


\section{FAULT DETECTION AND ISOLATION}

One of the main features of sliding-mode observers is the possibility of unknown input identification using the equivalent output injection (see, for example [40] and [41]). Here, the equivalent output injection will be used as the residual to achieve fault detection.

Define the output estimation error as

$$
\left\{\bar{y}_{\lambda_{i}}^{(j-1)}(t)-y_{i}{ }^{(j-1)}(t)\right\}_{i=1, j=1}^{p, r_{i}}=\left\{\epsilon_{\lambda_{i}, j}(t)\right\}_{i=1, j=1}^{p, r_{i}}, \quad \forall \lambda \in \Lambda
$$

Suppose that the active scenario is the fault-free case and therefore, the output error dynamics between system (9) and observer $\lambda=0$ is given by

$$
\begin{aligned}
& \dot{\epsilon}_{0_{1}, 1}=\epsilon_{0_{1}, 2}-\alpha_{1,1} M_{0_{1}}^{\frac{1}{r_{1}}}\left\lceil\epsilon_{0_{1}, 1}-v_{1}(t)\right\rfloor^{\frac{r_{1}-1}{r_{1}}}, \\
& \dot{\epsilon}_{0_{1}, j}=\epsilon_{0_{1}, j+1}-\alpha_{1, j} M_{0_{1}}^{\frac{1}{r_{1}-j+1}}\left\lceil\epsilon_{0_{1}, j}-\dot{\epsilon}_{0_{1}, j-1}\right\rfloor^{\frac{r_{1}-j}{r_{1}-j+1}}, j=2, \ldots, r_{1}-1, \\
& \dot{\epsilon}_{0_{1}, r_{1}}=L_{f\left(\bar{x}_{0}\right)}^{r_{1}} h_{1}\left(\bar{x}_{0}\right)-L_{f(x)}^{r_{1}} h_{1}(x)-\alpha_{1, r_{1}} M_{0_{1}}\left\lceil\epsilon_{0_{1}, r_{1}}-\dot{\epsilon}_{0_{1}, r_{1}-1}\right\rfloor^{0}, \\
& \quad \vdots \\
& \dot{\epsilon}_{0_{p}, 1}=\epsilon_{0_{p}, 2}-\alpha_{p, 1} M_{0_{p}}^{\frac{1}{r_{p}}}\left\lceil\epsilon_{0_{p}, 1}-v_{p}(t)\right\rfloor^{\frac{r_{p}-1}{r_{p}}}, \\
& \dot{\epsilon}_{0_{p}, j}=\epsilon_{0_{p}, j+1}-\alpha_{p, j} M_{0_{p}}^{\frac{1}{r_{p}-j+1}}\left\lceil\epsilon_{0_{p}, j}-\dot{\epsilon}_{0_{p}, j-1}\right\rfloor^{\frac{r_{p}-j}{r_{p}-j+1}}, j=2, \ldots, r_{p}-1, \\
& \dot{\epsilon}_{0_{p}, r_{p}}=L_{f\left(\bar{x}_{0}\right)}^{r_{p}} h_{p}\left(\bar{x}_{0}\right)-L_{f(x)}^{r_{p}} h_{p}(x)-\alpha_{p, r_{p}} M_{0_{p}}\left\lceil\epsilon_{0_{p}, r_{p}}-\dot{\epsilon}_{0_{p}, r_{p}-1}\right\rfloor^{0} .
\end{aligned}
$$

\section{Remark 7}

Notice that the output error dynamics between system (9) and the other observers do not necessarily have any particular structure. However, if the observability mapping is the same for every $\lambda$, the output error dynamics will have the same structure as (29). Nevertheless, the terms $\left\{L_{f\left(\bar{x}_{\lambda}\right)}^{r_{i}} h_{i}\left(\bar{x}_{\lambda}\right)-L_{f(x)}^{r_{i}} h_{i}(x)\right\}_{i=1}^{p}$ will be different, for all $\lambda \neq 0$, due to the system structure.

In steady state, all the terms $\left\{\epsilon_{0_{i}, r_{i}}(t)\right\}_{i=1}^{p}$ are directly affected by the discontinuous correction terms $\left\{\nu_{0_{i}, r_{i}}(t)\right\}_{i=1}^{p}=\left\{\left\lceil\epsilon_{0_{i}, r_{i}}-\dot{\epsilon}_{0_{i}, r_{i}-1}\right\rfloor^{0}\right\}_{i=1}^{p}$, i.e. they are zero in the "average" sense. Therefore, the equivalent output injection principle can be exploited.

The expressions for $\left\{\epsilon_{0_{i}, r_{i}}(t)\right\}_{i=1}^{p}$ are given by

$$
\begin{aligned}
\dot{\epsilon}_{0_{1}, r_{1}} & =L_{f\left(\bar{x}_{0}\right)}^{r_{1}} h_{1}\left(\bar{x}_{0}\right)-L_{f(x)}^{r_{1}} h_{1}(x)-\alpha_{1, r_{1}} M_{0_{1}} \nu_{0_{1}, r_{1}}(t), \\
\dot{\epsilon}_{0_{2}, r_{2}} & =L_{f\left(\bar{x}_{0}\right)}^{r_{2}} h_{2}\left(\bar{x}_{0}\right)-L_{f(x)}^{r_{2}} h_{2}(x)-\alpha_{2, r_{2}} M_{0_{2}} \nu_{0_{2}, r_{2}}(t), \\
\vdots & \\
\dot{\epsilon}_{0_{p}, r_{p}} & =L_{f\left(\bar{x}_{0}\right)}^{r_{p}} h_{p}\left(\bar{x}_{0}\right)-L_{f(x)}^{r_{p}} h_{p}(x)-\alpha_{p, r_{p}} M_{0_{p}} \nu_{0_{p}, r_{p}}(t) .
\end{aligned}
$$

At the moment in which the state estimation is reached, the discontinuous terms $\left\{\nu_{0_{i}, r_{i}}(t)\right\}_{i=1}^{p}$ take the value of the equivalent output injection, and, for the noise-free case; they are equal to zero, 
i.e.

$$
\begin{aligned}
0= & -\alpha_{1, r_{1}} M_{0_{1}} \nu_{0_{1}, r_{1}}^{e q}(t), \\
0= & -\alpha_{2, r_{2}} M_{0_{2}} \nu_{0_{2}, r_{2}}^{e q}(t), \\
& \vdots \\
0= & -\alpha_{p, r_{p}} M_{0_{p}} \nu_{0_{p}, r_{p}}^{e q}(t) .
\end{aligned}
$$

In this work, the functions $\nu_{0_{i}, r_{i}}^{e q}(t)$ in (31), which need to be applied to dynamics (29) after reaching the sliding surfaces $\epsilon_{i, r_{i}}(t)=0$, to ensure that the output error trajectories stays on the surfaces thereafter, are called the equivalent output injections (for details, see [42]).

The following equivalent output injection properties can be established:

1. The equivalent output injections describe the "average" effect of the high-frequency terms $\left\{\left\lceil\epsilon_{0_{i}, r_{i}}-\dot{\epsilon}_{0_{i}, r_{i}-1}\right\rfloor^{0}\right\}_{i=1}^{p}$.

2. The equivalent output injections provide estimations of the "disturbance" terms $\left\{L_{f\left(\bar{x}_{0}\right)}^{r_{i}} h_{i}\left(\bar{x}_{0}\right)-L_{f(x)}^{r_{i}} h_{i}(x)\right\}_{i=1}^{p}$.

Hence, taking into account the effect of noise and the statements of Theorem 1, equation (31) simplifies to

$$
\begin{gathered}
\gamma_{0_{1}, r_{1}+1} M_{0_{1}} \xi^{2} \geq\left|\alpha_{1, r_{1}} M_{0_{1}} \nu_{0_{1}, r_{1}}^{e q}(t)\right|, \\
\gamma_{0_{2}, r_{2}+1} M_{0_{2}} \xi^{2} \geq\left|\alpha_{2, r_{2}} M_{0_{2}} \nu_{0_{2}, r_{2}}^{e q}(t)\right|, \\
\quad \vdots \\
\gamma_{0_{p}, r_{p}+1} M_{0_{p}} \xi^{2} \geq\left|\alpha_{p, r_{p}} M_{0_{p}} \nu_{0_{p}, r_{p}}^{e q}(t)\right| .
\end{gathered}
$$

Then, the equivalent output injections satisfy the following inequalities

$$
\begin{gathered}
\left|\nu_{0_{1}, r_{1}}^{e q}(t)\right| \leq \frac{\gamma_{0_{1}, r_{1}+1}}{\alpha_{1, r_{1}}} \xi^{2}, \quad \forall t>t^{*} \\
\left|\nu_{0_{2}, r_{2}}^{e q}(t)\right| \leq \frac{\gamma_{0_{2}, r_{2}+1}}{\alpha_{2, r_{2}}} \xi^{2}, \quad \forall t>t^{*} \\
\vdots \\
\left|\nu_{0_{p}, r_{p}}^{e q}(t)\right| \leq \frac{\gamma_{0_{p}, r_{p}+1}}{\alpha_{p, r_{p}}} \xi^{2}, \quad \forall t>t^{*} .
\end{gathered}
$$

In accordance with the previous explanations, only the equivalent output injection of observer $\lambda=0$ will satisfy the inequalities (33). In general, the output error dynamics generated by the observers associated with $\lambda \neq 0$ will not have the structure (29), because the vector relative degree could change between the different scenarios, and Assumption 2, 3 and 4 may be only satisfied for the respective scenario. Consequently, the trajectories that are associated with the observers $\lambda \neq 0$ will be different from those that the system produces in the active scenario; thus, the equivalent output injection will not satisfy (33).

In particular, when the output error dynamics of another observer, e.g. observer $\lambda=1$, has the structure (29), both observers $\lambda=0$ and $\lambda=1$ have the same observability mapping. Hence, the 
expressions for $\left\{\epsilon_{1_{i}, r_{i}}(t)\right\}_{i=1}^{p}$ will be given by

$$
\begin{aligned}
\dot{\epsilon}_{1_{1}, r_{1}} & =L_{f\left(\bar{x}_{1}\right)}^{r_{1}} h_{1}\left(\bar{x}_{1}\right)-L_{f(x)}^{r_{1}} h_{1}(x)-\alpha_{1, r_{1}} M_{1_{1}} \nu_{1_{1}, r_{1}}(t), \\
\dot{\epsilon}_{1_{2}, r_{2}} & =L_{f\left(\bar{x}_{1}\right)}^{r_{2}} h_{2}\left(\bar{x}_{1}\right)-L_{f(x)}^{r_{2}} h_{2}(x)-\alpha_{2, r_{2}} M_{1_{2}} \nu_{1_{2}, r_{2}}(t), \\
\vdots & \\
\dot{\epsilon}_{1_{p}, r_{p}} & =L_{f\left(\bar{x}_{1}\right)}^{r_{p}} h_{p}\left(\bar{x}_{1}\right)-L_{f(x)}^{r_{p}} h_{p}(x)-\alpha_{p, r_{p}} M_{1_{p}} \nu_{1_{p}, r_{p}}(t) .
\end{aligned}
$$

If the gains $\left\{M_{1_{i}}\right\}_{i=1}^{p}$ are designed such that $\left\{L_{f\left(\bar{x}_{1}\right)}^{r_{i}} h_{i}\left(\bar{x}_{1}\right)-L_{f(x)}^{r_{i}} h_{i}(x)\right\}_{i=1}^{p} \leq\left\{M_{1_{i}}\right\}_{i=1}^{p}$ is satisfied, then the observer associated with $\lambda=1$ will estimate the state with an error proportional to a certain order of the noise. In this way, (34) can be rewritten as follows

$$
\begin{gathered}
\gamma_{1_{1}, r_{1}+1} M_{1_{1}} \xi^{2} \geq \underbrace{\left|L_{f\left(\bar{x}_{1}\right)}^{r_{1}} h_{1}\left(\bar{x}_{1}\right)-L_{f(x)}^{r_{1}} h_{1}(x)\right|}_{\left|\varepsilon_{1_{1}}\right|}+\left|\alpha_{1, r_{1}} M_{1_{1}} \nu_{1_{1}, r_{1}}^{e q}(t)\right|, \\
\gamma_{1_{2}, r_{2}+1} M_{1_{2}} \xi^{2} \geq \underbrace{\left|L_{f\left(\bar{x}_{1}\right)}^{r_{2}} h_{2}\left(\bar{x}_{1}\right)-L_{f(x)}^{r_{2}} h_{2}(x)\right|}_{\left|\varepsilon_{1_{2}}\right|}+\left|\alpha_{2, r_{2}} M_{1_{2}} \nu_{1_{2}, r_{2}}^{e q}(t)\right|, \\
\vdots \\
\gamma_{1_{p}, r_{p}+1} M_{1_{p}} \xi^{2} \geq \underbrace{\left|L_{f\left(\bar{x}_{1}\right)}^{r_{p}} h_{p}\left(\bar{x}_{1}\right)-L_{f(x)}^{r_{p}} h_{p}(x)\right|}_{\left|\varepsilon_{1_{p}}\right|}+\left|\alpha_{p, r_{p}} M_{1_{p}} \nu_{1_{p}, r_{p}}^{e q}(t)\right|,
\end{gathered}
$$

where $\left\{\varepsilon_{1_{i}}\right\}_{i=1}^{p}$ are the differences in the model corresponding to the observer associated with $\lambda=1$. The following lemma establishes the class of faults that can be detected and isolated for the particular case in which the observability mappings are equal for two different values of $\lambda$.

\section{Lemma 2}

Let the bank of HOSM observers (27) be applied to the system (1)-(3). For all $\eta \neq \sigma$ such that $\eta, \sigma \in$ $\Lambda$; with the property that their corresponding observability mappings are the same, if the trajectories $\left\{\varepsilon_{\eta_{i}}\right\}_{i=1}^{p}$ and $\left\{\varepsilon_{\sigma_{i}}\right\}_{i=1}^{p}$ are sufficiently large out of their active scenario; then the faults $\eta$ and $\sigma$ can be detected and isolated by means of $\nu_{\eta}^{e q}=\left[\nu_{\eta_{1}, r_{1}}^{e q}, \cdots, \nu_{\eta_{p}, r_{p}}^{e q}\right]^{T}$ and $\nu_{\sigma}^{e q}=\left[\nu_{\sigma_{1}, r_{1}}^{e q}, \cdots, \nu_{\sigma_{p}, r_{p}}^{e q}\right]^{T}$.

\section{Proof}

It is clear that if the scenarios $\eta$ and $\sigma$ have the same observability mapping, they will have the structure (29), and the only difference will be given by the terms $\left\{\varepsilon_{\eta_{i}}\right\}_{i=1}^{p}$ and $\left\{\varepsilon_{\sigma_{i}}\right\}_{i=1}^{p}$. Then, if the difference between the models is sufficiently large out of their active scenario, then, supposing 
that the active scenario is $\eta$, the equivalent output injections satisfy the following inequalities

$$
\begin{aligned}
& \left|\nu_{\sigma_{1}, r_{1}}^{e q}(t)\right| \leq \frac{\gamma_{\eta_{1}, r_{1}+1}}{\alpha_{1, r_{1}}} \xi^{2}<\frac{\gamma_{\sigma_{1}, r_{1}+1}}{\alpha_{1, r_{1}}} \xi^{2}+\frac{1}{\alpha_{1, r_{1}} M_{1_{1}}}\left|\varepsilon_{\sigma_{1}}\right|, \\
& \left|\nu_{\sigma_{2}, r_{2}}^{e q}(t)\right| \leq \frac{\gamma_{\eta_{2}, r_{2}+1}}{\alpha_{2, r_{2}}} \xi^{2}<\frac{\gamma_{\sigma_{2}, r_{2}+1}}{\alpha_{2, r_{2}}} \xi^{2}+\frac{1}{\alpha_{2, r_{2}} M_{1_{2}}}\left|\varepsilon_{\sigma_{2}}\right|, \\
& \quad \vdots \\
& \left|\nu_{\sigma_{p}, r_{p}}^{e q}(t)\right| \leq \frac{\gamma_{\eta_{p}, r_{p}+1}}{\alpha_{p, r_{p}}} \xi^{2}<\frac{\gamma_{\sigma_{p}, r_{p}+1}}{\alpha_{p, r_{p}}} \xi^{2}+\frac{1}{\alpha_{p, r_{p}} M_{1_{p}}}\left|\varepsilon_{\sigma_{p}}\right| .
\end{aligned}
$$

Notice that the terms that depend on the amplitude of the noise have the same order, i.e. $O\left(\xi^{2}\right)$, thus, the order of the terms $\left\{\varepsilon_{\sigma_{i}}\right\}_{i=1}^{p}$ has to be discernably larger in order to detect and isolate the faults $\eta$ and $\sigma$. In this way, the faults will be distinguishable using the information of $\nu_{\eta}^{e q}$ and $\nu_{\sigma}^{e q}$.

In the other case, when the observability mappings are different, inequalities (36) are satisfied trivially.

Now, the relations (33) will be exploited to establish a residual evaluation. The overall power of each element of the equivalent output injections will be taken as a scalar residual, i.e.

$$
\left\{r_{\lambda}(t)\right\}_{\lambda=0}^{q}=\left\{\nu_{\lambda_{1}, r_{1}}^{e q}(t)^{2}+\nu_{\lambda_{2}, r_{2}}^{e q}(t)^{2}+\cdots+\nu_{\lambda_{p}, r_{p}}^{e q}(t)^{2}\right\}_{\lambda=0}^{q} .
$$

Theoretically, if condition (36) is satisfied under statements given by Lemma 2 or for different observability mappings, a simple threshold-based decision criterion could be applied to the signals $r_{\lambda}(t)$ in order to achieve fault detection. Such a detection rule could be represented as follows:

$$
\hat{\lambda}=\arg \min _{\lambda} r_{\lambda}(t) .
$$

However, the signals $r_{\lambda}(t), \lambda \neq \lambda^{*}$, can occasionally cross the zero value when the active scenario is $\lambda^{*}$, which would make the decision (38) unreliable. Therefore, due to the previous explanation and to measurement noise, the average power of the signals $r_{\lambda}(t)$ is taken over a suitable recedinghorizon time interval of finite length, i.e.

$$
\left\{R_{\lambda}(t)\right\}_{\lambda=0}^{q}=\left\{\frac{1}{\Delta T} \int_{t-\Delta T}^{t} r_{\lambda}(\tau) d \tau\right\}_{\lambda=0}^{q},
$$

where $\Delta T$ is the width of the time interval used ${ }^{\dagger \dagger}$. Then, the Fault Detection Rule (FDR) is established as the value of $\lambda$ for which $R_{\lambda}(t)$ is minimum, i.e.

$$
\hat{\lambda}=\arg \min _{\lambda} R_{\lambda}(t)
$$

with $\hat{\lambda}$ representing the active scenario. Therefore, it is possible to detect and isolate the fault in the system according to the residuals from each observer. Obviously, each residual is sensitive, i.e.

${ }^{\dagger \dagger}$ The width $\Delta T$ varies according to the kind of system, to the faults or even to the inputs. However, it can be defined as the minimum time interval for which (40) has a unique $\hat{\lambda}$ for all possible $\lambda$. 
satisfies condition (33) or (36), only to the corresponding fault. Hence, it is possible to distinguish between the occurrence of each fault due to the characteristics of the equivalent output injection.

\section{Remark 8}

Notice that it will only be possible to detect and isolate faults just after the observer for the active scenario has converged, i.e. after time $t^{*}, t_{f_{\Delta, \kappa}}^{*}$ or $t_{B_{\Delta, \kappa}}^{*}$, depending on the case.

Theoretically, the equivalent output injection is the result of infinite switching frequency in the discontinuous term $\nu_{\lambda}$. However, the realization of the observer produces finite high frequency switching, making necessary the application of a filter.

Define the following equivalent output injection estimator of $\nu_{\lambda_{\text {eq }}}$ :

$$
\tau_{\lambda} \dot{\bar{\nu}}_{\lambda_{\mathrm{eq}}}=\nu_{\lambda}-\bar{\nu}_{\lambda_{\mathrm{eq}}}
$$

where each $\tau_{\lambda}$ is designed according to [43], i.e. $\tau_{s} \ll \tau_{\lambda} \ll 1$ for all $\lambda=0, \ldots, q$, where $\tau_{s}$ is the sample time. One possible choice is $\tau_{\lambda}=\sqrt{\tau_{s}}$. Then, the equivalent output injections can be used to detect and isolate the faults.

Notice that the filter in (41) can be designed to estimate the equivalent output injection and also to avoid to a certain extent the effect of noise (as is illustrated in the simulations in the sequel).

\subsection{Discussion: Faults not belonging to $\mathcal{F}$ and Uncertainties}

For the case of a fault not belonging to $\mathcal{F}$, if it satisfies the relative degree condition (4), and all the assumptions for the plant or actuator fault case, such an observer will be able to estimate the states. However in this case, every residual may be influenced by such a fault, indicating that it is not contained in $\mathcal{F}$. In this way fault detection may still be achievable, but not isolation.

If the new fault does not satisfy the relative degree condition in (4), for a plant or actuator fault, no observer will estimate the state. However, the fault detection may still be possible due to the properties of the equivalent output injection, again indicating that such a fault is not contained in the fault set $\mathcal{F}$.

For the uncertainty case, a detailed study is formally necessary since the uncertainties directly affect, and possibly in different forms, the proposed residual. Also, the process for the residual design would have to change drastically or it would be necessary to undertake a study, for example a statistical one; of the uncertainty effects on the residuals, and thus, to establish some thresholds that allow to realize the fault detection and isolation process. However, in order to show that the proposed approach can work under certain uncertainties, simulation results are shown using exactly the same approach described in this section.

\section{SIMULATION EXAMPLE}

Consider the mass-spring-damper system, depicted in Fig. 1. 


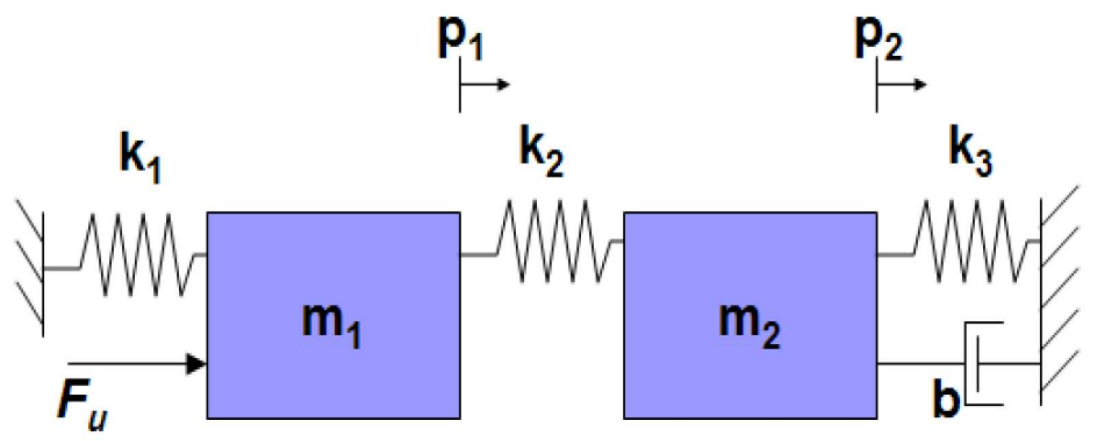

Figure 1. Block diagram of mass-spring-damper system.

The set of differential equations that describe the system behavior are the following:

$$
\begin{aligned}
& m_{1} \ddot{p}_{1}+\left(k_{1}+k_{2}\right) p_{1}-k_{2} p_{2}=F_{u}, \\
& m_{2} \ddot{p}_{2}+\left(k_{2}+k_{3}\right) p_{2}+b \tanh \left(\dot{p}_{2}\right)-k_{2} p_{1}=0,
\end{aligned}
$$

where $p_{1}$ and $p_{2}$ describe the positions, $F_{u}$ is the input force acting on the system, $m_{1}$ and $m_{2}$ are the masses, $k_{1}, k_{2}$ and $k_{3}$ represent the spring constants, and $b$ is the dry friction coefficient. Then, system (42)-(43) can be represented in the form of (9) as follows:

$$
\begin{aligned}
& {\left[\begin{array}{l}
\dot{x}_{1} \\
\dot{x}_{2} \\
\dot{x}_{3} \\
\dot{x}_{4}
\end{array}\right]=\left[\begin{array}{c}
x_{2} \\
-\frac{k_{1}+k_{2}}{m_{1}} x_{1}+\frac{k_{2}}{m_{1}} x_{3} \\
x_{4} \\
\frac{k_{2}}{m_{2}} x_{1}-\frac{k_{2}+k_{3}}{m_{2}} x_{3}-\frac{b}{m_{2}} \tanh \left(x_{4}\right)
\end{array}\right]+\left[\begin{array}{c}
0 \\
\frac{1}{m_{1}} \\
0 \\
0
\end{array}\right] u,} \\
& y=\left[\begin{array}{ll}
x_{1} & x_{3}
\end{array}\right]^{T},
\end{aligned}
$$

where $x=\left[\begin{array}{llll}p_{1} & \dot{p}_{1} & p_{2} & \dot{p}_{2}\end{array}\right]^{T}$ is the state vector, $u=F_{u}$ is the input force, and $y$ the measurable output. The parameters of the mass-springer-damper system are shown in Table I.

Table I. Mass-springer-damper parameters

\begin{tabular}{c||c}
\hline \hline Parameter & Value \\
\hline$m_{1}$ & $1.28[\mathrm{~kg}]$ \\
$m_{2}$ & $1.05[\mathrm{~kg}]$ \\
$k_{1}$ & $450[\mathrm{~N} / \mathrm{m}]$ \\
$k_{2}$ & $175[\mathrm{~N} / \mathrm{m}]$ \\
$k_{3}$ & $450[\mathrm{~N} / \mathrm{m}]$ \\
$b$ & $15[\mathrm{Ns} / \mathrm{m}]$ \\
\hline \hline
\end{tabular}

Simulations have been done in the MATLAB Simulink environment, with the Euler discretization method, a sampling time $\tau_{s}=0.001[\mathrm{sec}]$, and the real initial conditions $x(0)=[0,0,0,0]^{T}$. In the following, state observers are designed to estimate the velocities. 


\subsection{Fault-Free Observer}

First, the fault-free observer $(\lambda=0)$ will be designed. The Jacobian matrix defined in (10) has the following structure:

$$
\frac{\partial \Phi_{0}\left(\bar{x}_{0}\right)}{\partial \bar{x}_{0}}=\left[\begin{array}{llll}
1 & 0 & 0 & 0 \\
0 & 1 & 0 & 0 \\
0 & 0 & 1 & 0 \\
0 & 0 & 0 & 1
\end{array}\right] .
$$

It is clear that $\operatorname{rank}\left(\frac{\partial \Phi_{0}\left(\bar{x}_{0}\right)}{\partial \bar{x}_{0}}\right)=4$ for all $\bar{x}_{0} \in \mathcal{X} \subseteq \Re^{4}$ and therefore Assumption 1 is satisfied. Then, the fault-free observer takes the following form

$$
\begin{aligned}
& {\left[\begin{array}{c}
\dot{\bar{x}}_{01} \\
\dot{\bar{x}}_{02} \\
\dot{\bar{x}}_{03} \\
\dot{\bar{x}}_{04}
\end{array}\right]=\left[\begin{array}{c}
\bar{x}_{02} \\
-\frac{k_{1}+k_{2}}{m_{1}} \bar{x}_{01}+\frac{k_{2}}{m_{1}} \bar{x}_{03} \\
\bar{x}_{04} \\
\frac{k_{2}}{m_{2}} \bar{x}_{01}-\frac{k_{2}+k_{3}}{m_{2}} \bar{x}_{03}-\frac{b}{m_{2}} \tanh \left(\bar{x}_{04}\right)
\end{array}\right]+\left[\begin{array}{c}
0 \\
\frac{1}{m_{1}} \\
0 \\
0
\end{array}\right] u+\left(\frac{\partial \Phi_{0}\left(\bar{x}_{0}\right)}{\partial \bar{x}_{0}}\right)^{-1} \nu_{0}} \\
& \bar{y}_{0}=\left[\begin{array}{ll}
\bar{x}_{01} & \bar{x}_{03}
\end{array}\right]^{T}
\end{aligned}
$$

with observer initial conditions $\bar{x}_{0}(0)=[0.1,0.1,0.1,0.1]^{T}$. The correction term is calculated using the following auxiliary dynamics:

$$
\begin{aligned}
& \dot{\vartheta}_{0_{1,1}}=\vartheta_{0_{1,2}}-\alpha_{1,1} M_{0_{1}}^{\frac{1}{2}}\left[e_{y_{0_{1}}}(t)\right\rfloor^{\frac{1}{2}}, \\
& \dot{\vartheta}_{0_{1,2}}=-\alpha_{1,2} M_{0_{1}}\left[\vartheta_{0_{1,2}}-\dot{\vartheta}_{0_{1,1}}\right\rfloor^{0}, \\
& \dot{\vartheta}_{0_{2,1}}=\vartheta_{2,2}-\alpha_{0_{2,1}} M_{0_{2}}^{\frac{1}{2}}\left[e_{y_{0_{2}}}(t)\right\rfloor^{\frac{1}{2}}, \\
& \dot{\vartheta}_{0_{2,2}}=-\alpha_{2,2} M_{0_{2}}\left[\vartheta_{0_{2,2}}-\dot{\vartheta}_{0_{2,1}}\right\rfloor^{0},
\end{aligned}
$$

where $e_{y_{0_{1}}}=\bar{x}_{01}-x_{1}, e_{y_{0_{2}}}=\bar{x}_{03}-x_{3}$, and the parameters $\alpha_{1,1}=\alpha_{2,1}=1.5, \alpha_{1,2}=\alpha_{2,2}=1.1$. Based on each acceleration, i.e. $\dot{x}_{2}$ and $\dot{x}_{4}$, and the initial condition error, the gains $M_{0_{1}}=1$ and $M_{0_{2}}=0.5$ are sufficiently large constants such that Assumption 2 is satisfied for each output. Then, the correction term takes the following form:

$$
\nu_{0}=\left[\begin{array}{c}
-\alpha_{1,1} M_{0_{1}}^{\frac{1}{2}}\left\lceil e_{y_{0_{1}}}(t)\right\rfloor^{\frac{1}{2}} \\
-\alpha_{1,2} M_{0_{1}}\left[\vartheta_{0_{1,2}}-\dot{\vartheta}_{0_{1,1}}\right\rfloor^{0} \\
-\alpha_{2,1} M_{0_{2}}^{\frac{1}{2}}\left\lceil e_{y_{0_{2}}}(t)\right\rfloor^{\frac{1}{2}} \\
-\alpha_{2,2} M_{0_{2}}\left[\vartheta_{0_{2,2}}-\dot{\vartheta}_{0_{2,1}}\right]^{0}
\end{array}\right] .
$$




\subsection{Actuator Fault Observer}

Consider the case when an actuator fault occurs in system (44)-(45) and the actuator is working at $10 \%$ of its capability, i.e. $F_{1}(x) \omega_{1}=B_{a_{1}} u$. Then, the dynamics has the following structure:

$$
\begin{aligned}
& {\left[\begin{array}{l}
\dot{x}_{1} \\
\dot{x}_{2} \\
\dot{x}_{3} \\
\dot{x}_{4}
\end{array}\right]=\left[\begin{array}{c}
x_{2} \\
-\frac{k_{1}+k_{2}}{m_{1}} x_{1}+\frac{k_{2}}{m_{1}} x_{3} \\
x_{4} \\
\frac{k_{2}}{m_{2}} x_{1}-\frac{k_{2}+k_{3}}{m_{2}} x_{3}-\frac{b}{m_{2}} \tanh \left(x_{4}\right)
\end{array}\right]+\left[\begin{array}{c}
0 \\
\frac{0.1}{m_{1}} \\
0 \\
0
\end{array}\right] u} \\
& y=\left[\begin{array}{ll}
x_{1} & x_{3}
\end{array}\right]^{T},
\end{aligned}
$$

The observability mapping for system (50)-(51) is the same as that for the system (44)-(45) and the Jacobian matrix takes the same structure, i.e. $\frac{\partial \Phi_{1}\left(\bar{x}_{1}\right)}{\partial \bar{x}_{1}}=\frac{\partial \Phi_{0}\left(\bar{x}_{0}\right)}{\partial \bar{x}_{0}}$.

Thus Assumption 1 is satisfied and the actuator fault observer takes the following form

$$
\begin{aligned}
& {\left[\begin{array}{c}
\dot{\bar{x}}_{11} \\
\dot{\bar{x}}_{12} \\
\dot{\bar{x}}_{13} \\
\dot{\bar{x}}_{14}
\end{array}\right]=\left[\begin{array}{c}
\bar{x}_{12} \\
-\frac{k_{1}+k_{2}}{m_{1}} \bar{x}_{11}+\frac{k_{2}}{m_{1}} \bar{x}_{13} \\
\bar{x}_{14} \\
\frac{k_{2}}{m_{2}} \bar{x}_{11}-\frac{k_{2}+k_{3}}{m_{2}} \bar{x}_{13}-\frac{b}{m_{2}} \tanh \left(\bar{x}_{14}\right)
\end{array}\right]+\left[\begin{array}{c}
0 \\
\frac{0.1}{m_{1}} \\
0 \\
0
\end{array}\right] u+\left(\frac{\partial \Phi_{1}\left(\bar{x}_{1}\right)}{\partial \bar{x}_{1}}\right)^{-1} \nu_{1}} \\
& \bar{y}_{1}=\left[\begin{array}{ll}
\bar{x}_{11} & \bar{x}_{13}
\end{array}\right]^{T}
\end{aligned}
$$

with observer initial conditions $\bar{x}_{1}(0)=[0.1,0.1,0.1,0.1]^{T}$. The correction term is calculated using the auxiliary dynamics in (48) replacing $e_{y_{01}}$ by $e_{y_{11}}=\bar{x}_{11}-x_{1}$ and $e_{y_{03}}$ by $e_{y_{13}}=\bar{x}_{13}-x_{3}$. Choosing $M_{1, f_{\Delta, 1}}=1$ and $M_{2, f_{\Delta, 1}}=0.5$ is sufficiently to ensure that Assumption 4 is satisfied for each output.

\subsection{Actuator + Plant Fault Observer}

Consider the case when a plant fault occurs in system (50)-(51) and the spring effect $k_{1}$ is no longer present, i.e. $F_{2}(x) \omega_{2}=B_{a_{1}} u+\Delta_{2} f_{2}(x)$. Then, the dynamics has the following structure:

$$
\begin{aligned}
& {\left[\begin{array}{l}
\dot{x}_{1} \\
\dot{x}_{2} \\
\dot{x}_{3} \\
\dot{x}_{4}
\end{array}\right]=\left[\begin{array}{c}
x_{2} \\
-\frac{k_{2}}{m_{1}} x_{1}+\frac{k_{2}}{m_{1}} x_{3} \\
x_{4} \\
\frac{k_{2}}{m_{2}} x_{1}-\frac{k_{2}+k_{3}}{m_{2}} x_{3}-\frac{b}{m_{2}} \tanh \left(x_{4}\right)
\end{array}\right]+\left[\begin{array}{c}
0 \\
\frac{0.1}{m_{1}} \\
0 \\
0
\end{array}\right] u,} \\
& y=\left[\begin{array}{ll}
x_{1} & x_{3}
\end{array}\right]^{T},
\end{aligned}
$$

Now, an actuator + plant fault observer $(\lambda=2)$ will be designed. The observability mapping for system (54)-(55) is the same as that for system (50)-(51). The Jacobian matrix also takes the same structure, i.e. $\frac{\partial \Phi_{2}\left(\bar{x}_{2}\right)}{\partial \bar{x}_{2}}=\frac{\partial \Phi_{1}\left(\bar{x}_{1}\right)}{\partial \bar{x}_{1}}$. 
Therefore, Assumption 1 is satisfied and the actuator + plant fault observer takes the following form

$$
\begin{aligned}
& {\left[\begin{array}{c}
\dot{\bar{x}}_{21} \\
\dot{\bar{x}}_{22} \\
\dot{\bar{x}}_{23} \\
\dot{\bar{x}}_{24}
\end{array}\right]=\left[\begin{array}{c}
\bar{x}_{22} \\
-\frac{k_{2}}{m_{1}} \bar{x}_{21}+\frac{k_{2}}{m_{1}} \bar{x}_{23} \\
\bar{x}_{24} \\
\frac{k_{2}}{m_{2}} \bar{x}_{21}-\frac{k_{2}+k_{3}}{m_{2}} \bar{x}_{23}-\frac{b}{m_{2}} \tanh \left(\bar{x}_{24}\right)
\end{array}\right]+\left[\begin{array}{c}
0 \\
\frac{0.1}{m_{1}} \\
0 \\
0
\end{array}\right] u+\left(\frac{\partial \Phi_{1}\left(\bar{x}_{2}\right)}{\partial \bar{x}_{2}}\right)^{-1} \nu_{2}} \\
& \bar{y}_{2}=\left[\begin{array}{ll}
\bar{x}_{21} & \bar{x}_{23}
\end{array}\right]^{T}
\end{aligned}
$$

with observer initial conditions $\bar{x}_{2}(0)=[0.1,0.1,0.1,0.1]^{T}$. The correction term is calculated using the auxiliary dynamics in (48) replacing $e_{y_{01}}$ by $e_{y_{21}}=\bar{x}_{21}-x_{1}$ and $e_{y_{03}}$ by $e_{y_{23}}=\bar{x}_{23}-x_{3}$. Choosing $M_{1, B_{\Delta, 2}}=1$ and $M_{2, B_{\Delta, 2}}=0.5$ is sufficiently large to ensure that Assumption 3 is satisfied for each output.

The parameter $\tau_{\lambda}$ is the same for every observer: specifically $\tau_{\lambda}=0.06$ for $\lambda=0,1,2$. The simulation develops in the following way: the system is fault-free during the first $5[\mathrm{sec}]$ after which the first actuator fault happens (whereby the actuator works at only $10 \%$ of its capability); then, at $10[\mathrm{sec}]$, the effect of spring 1 is removed when the actuator + plant fault occurs. The results are depicted in Figs. 2-5.

The residuals generated by the observers can be seen in Fig. 3. It is clear that only one residual is almost equal to zero when the corresponding fault is acting on the system. Therefore, the fault will be detected and isolated implementing the FDR scheme given in (38) with $\Delta T=0.01$. The corresponding results are shown in graph $c$ ) of Fig. 3. Finally, in graphs $d$ ) and $e$ ), a zoom at the moment when the Actuator + Plant fault has occurred is shown. It is possible to see that there exists a time delay $\left(t_{d} \approx 0.025[\mathrm{sec}]\right)$ after the fault occurs. Of course, this time delay is due to the filter used to estimate the equivalent output injection. However, this time could be arbitrarily reduced by taking different values for the parameter $\tau_{\lambda}$.

Next the same simulation scenario is repeated, taking into account the fact that the output contains measurement noise (see Fig. 4).

The noisy residuals generated by the observers can be seen in Fig. 5. In graphs $a$ ), b) and $c$ ) the effect of the noise on the residuals is shown using the same filter parameter $\tau_{\lambda}$ as before. It is clear that the noise affects the FDR producing false alarms (see graph $c$ )). Nevertheless, the performance can be improved if the filter parameter $\tau_{\lambda}$ is modified taking into account some noise information (shown in graphs $d$ ), e) and $f$ )).

In order to show that the proposed approach is robust again certain uncertainties, consider the following uncertainty mass-spring-damper system:

$$
\left[\begin{array}{c}
\dot{x}_{1} \\
\dot{x}_{2} \\
\dot{x}_{3} \\
\dot{x}_{4}
\end{array}\right]=\left[\begin{array}{c}
x_{2} \\
-\frac{k_{1}+k_{2}}{m_{1}} x_{1}+\frac{k_{2}}{m_{1}} x_{3} \\
x_{4} \\
\frac{k_{2}}{m_{2}} x_{1}-\frac{k_{2}+k_{3}}{m_{2}} x_{3}-\frac{b}{m_{2}} \tanh \left(x_{4}\right)
\end{array}\right]+\left[\begin{array}{c}
0 \\
\frac{1}{m_{1}} \\
0 \\
0
\end{array}\right] u+\left[\begin{array}{cccc}
0 & 0 & 0 & 0 \\
20 & 0 & 10 & 0 \\
0 & 0 & 0 & 0 \\
10 & 0 & 20 & 2
\end{array}\right] x,
$$



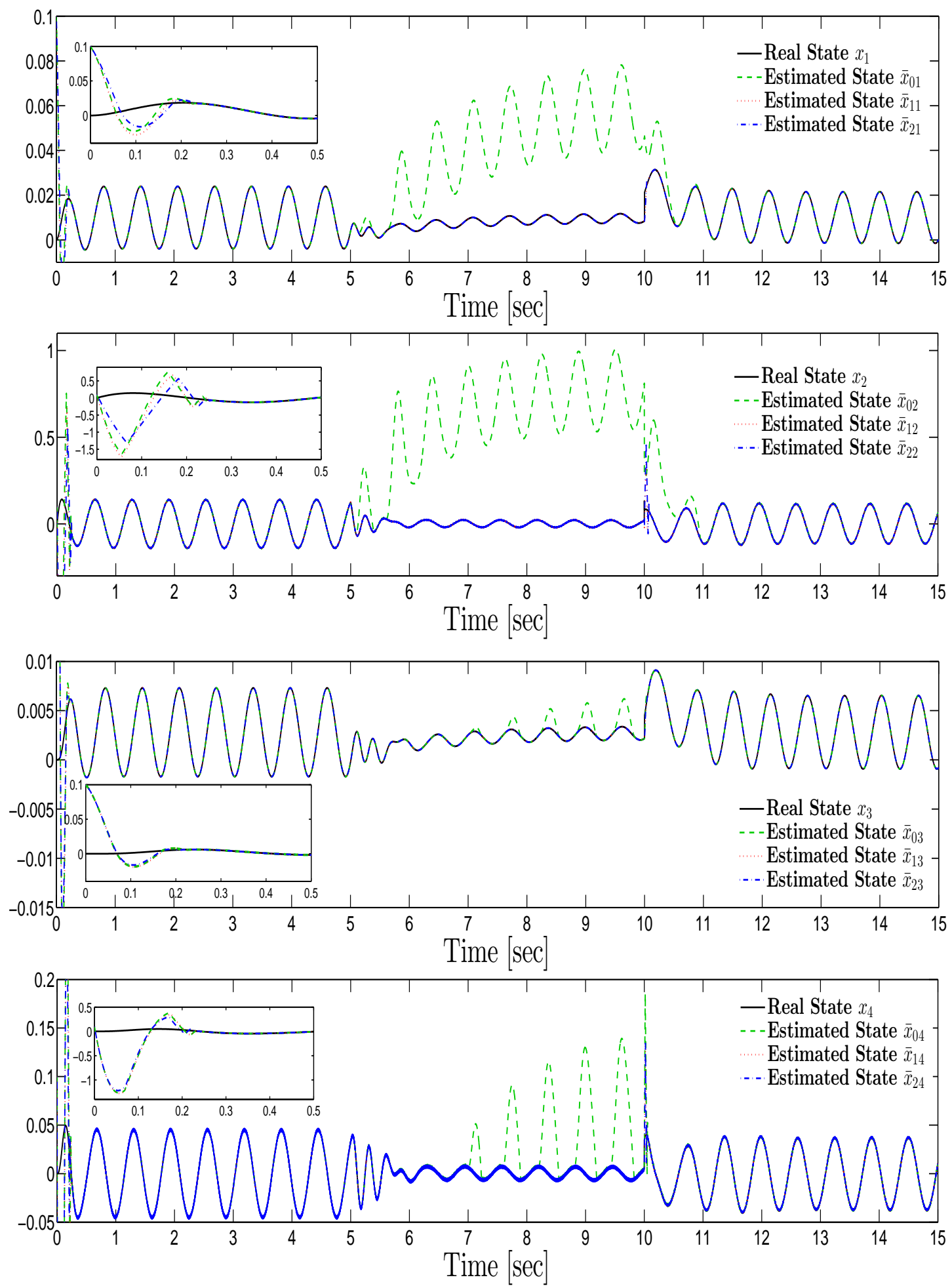

Figure 2. Real and State Estimation Trajectories. It is clear that each observer estimates its state in the corresponding scenario. Nevertheless, observers $\lambda=1$ and $\lambda=2$ clearly maintain the estimation in spite of the fault occurrence, while observer $\lambda=0$ loses accuracy of estimation when an actuator fault happens due to the corresponding gain $M_{1}$ is not able to support such a fault. 


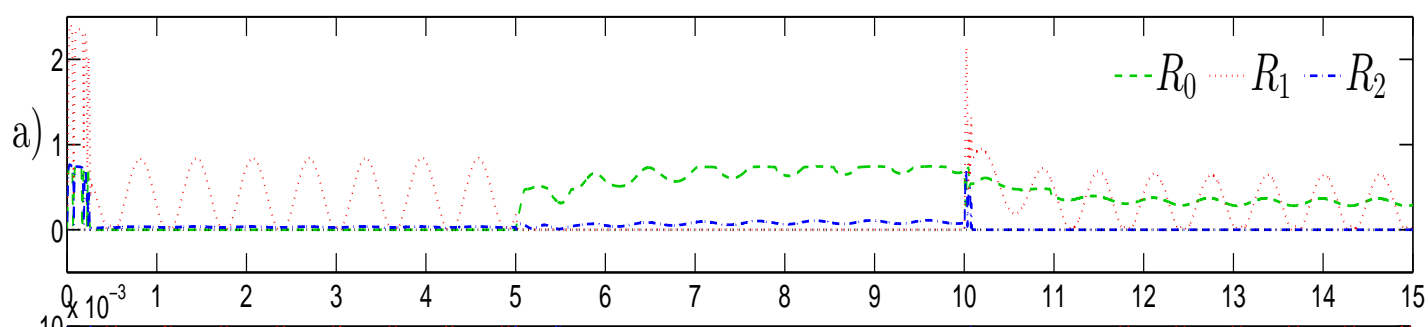

b)
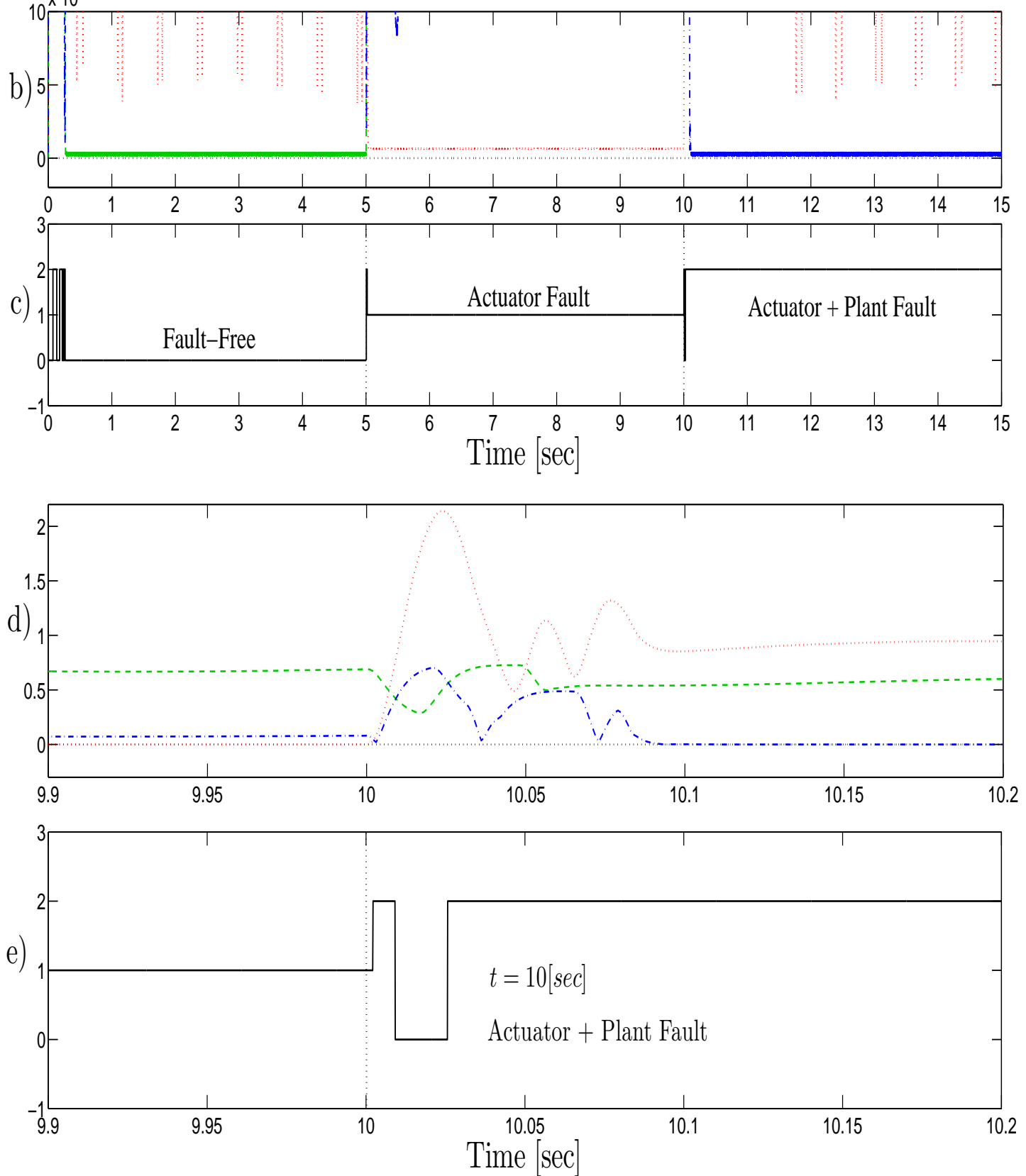

Figure 3. Residuals and Fault Detection Rule. a) Residuals $R_{0}, R_{1}$ and $R_{2}$. b) A zoom in a zero neighborhood of the residuals. c) Fault detection rule ( $\lambda$ estimation). d) Zoom at the moment of Actuator + Plant Fault in the residuals. e) Zoom at the moment of the Actuator + Plant Fault in the FDR. 

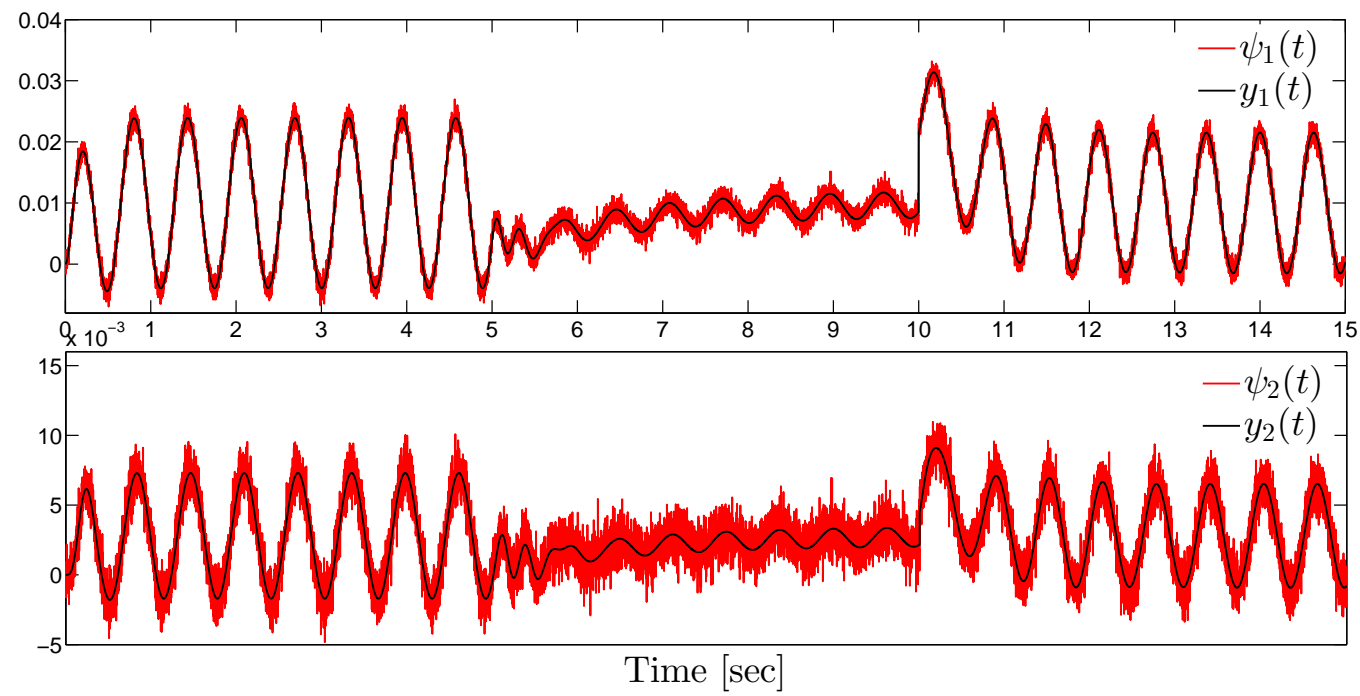

Figure 4. Output with and without Measurement Noise. The noisy signal is generated by a sum of sinusoidal signals of high frequency and amplitude of $3 \times 10^{-3}$.

where the last term represents parameter uncertainty. The simulation is developing in the same way which was described previously. For this simulation the observer gains are chosen as follows: $M_{0_{1}}=10$ and $M_{0_{2}}=5$ for the fault-free; $M_{1, f_{\Delta, 1}}=10$ and $M_{2, f_{\Delta, 1}}=5$, for the actuator fault; $M_{1, B_{\Delta, 2}}=10$ and $M_{2, B_{\Delta, 2}}=5$ for the actuator + plant fault, and the filter constant $\tau_{\lambda}=0.06$ for each observer. Then, the FDR (38) is implemented with $\Delta T=0.1$. The corresponding results are depicted by Fig. 6. It is easy to see that even in the uncertainty case it is still possible to solve the FDI problem with the proposed approach. Notice that may exist such uncertainties, high enough, that spoil the proposed approach. Therefore, it is necessary to realize a more detailed study of the FDI problem under uncertainties.

\section{CONCLUSIONS}

A combination of HOSM observers and a multiple-model approach is proposed to solve the FDI problem for a certain class of nonlinear systems. Under structural conditions, and based on the main features of sliding-mode observers, the value of the equivalent injection is used as a residual generator for detecting particular faults acting on the system. The isolation problem is solved using a multiple-model approach. The fast convergence of the HOSM methods provide fast FDI. The effect of Lebesgue-measurable noise in the outputs has been studied and their corresponding effects were described. The workability of the proposed methodology has been illustrated in simulations.

\section{REFERENCES}

1. Ríos H, Edwards C, Davila J, Fridman L. Fault detection and isolation for nonlinear systems via hosm multiple observer. Proceedings of the 8th IFAC Symposium on Fault Detection, Supervision and Safety of Technical Processes, Mexico City, Mexico, 2012; 534-539. 

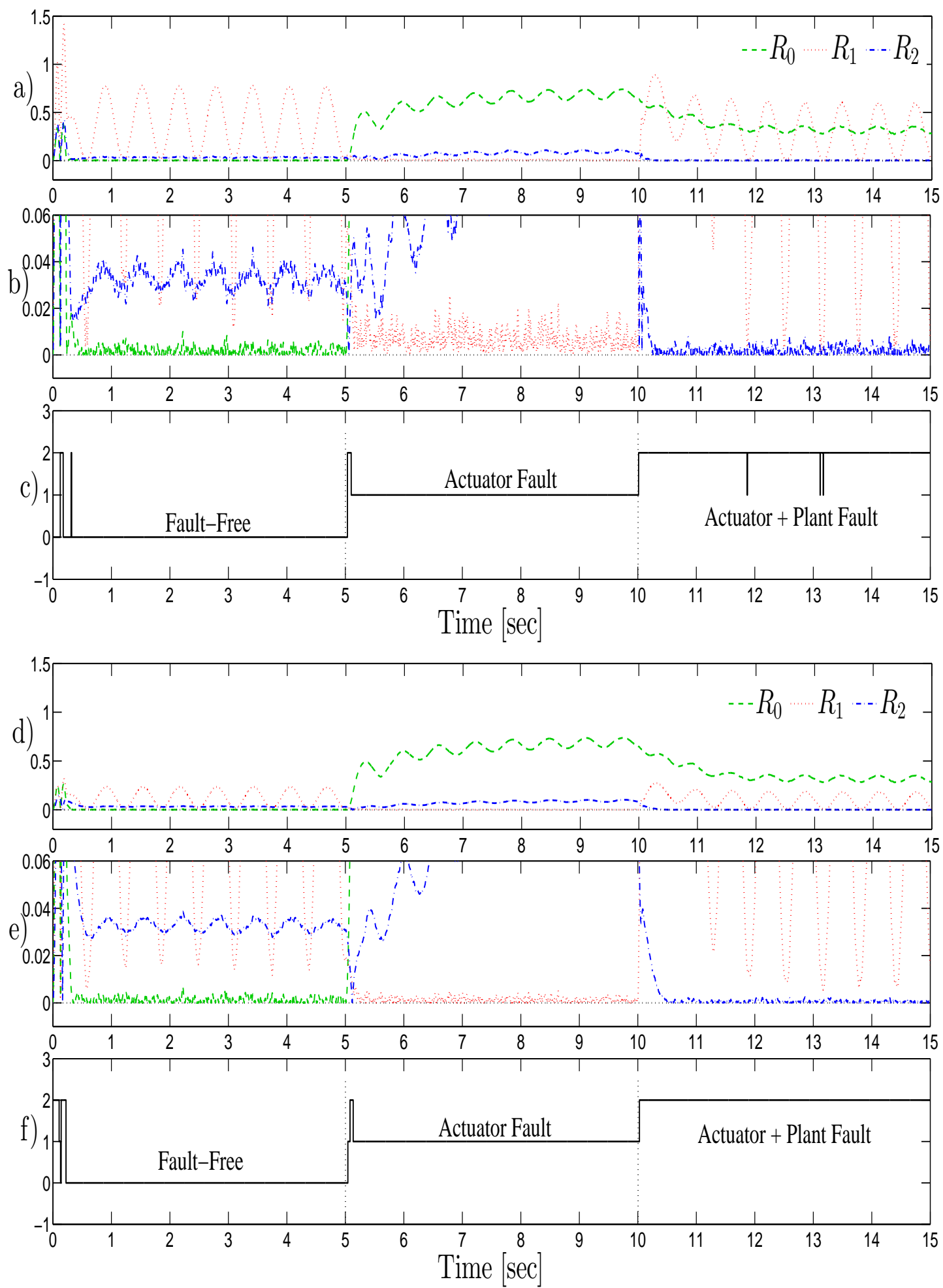

Figure 5. Residuals and Fault Detection Rule with Noise. a) Residuals $R_{0}, R_{1}$ and $R_{2}, \tau_{\lambda}=0.06$. b) $\mathrm{A}$ zoom in a zero neighborhood of the residuals, $\tau_{\lambda}=0.06$. c) Fault detection rule, $\tau_{\lambda}=0.06$. $d$ ) Residuals $R_{0}, R_{1}$ and $R_{2}, \tau_{\lambda}=0.0775$. e) A zoom in a zero neighborhood of the residuals, $\tau_{\lambda}=0.0775 . f$ ) Fault detection rule, $\tau_{\lambda}=0.0775$. 

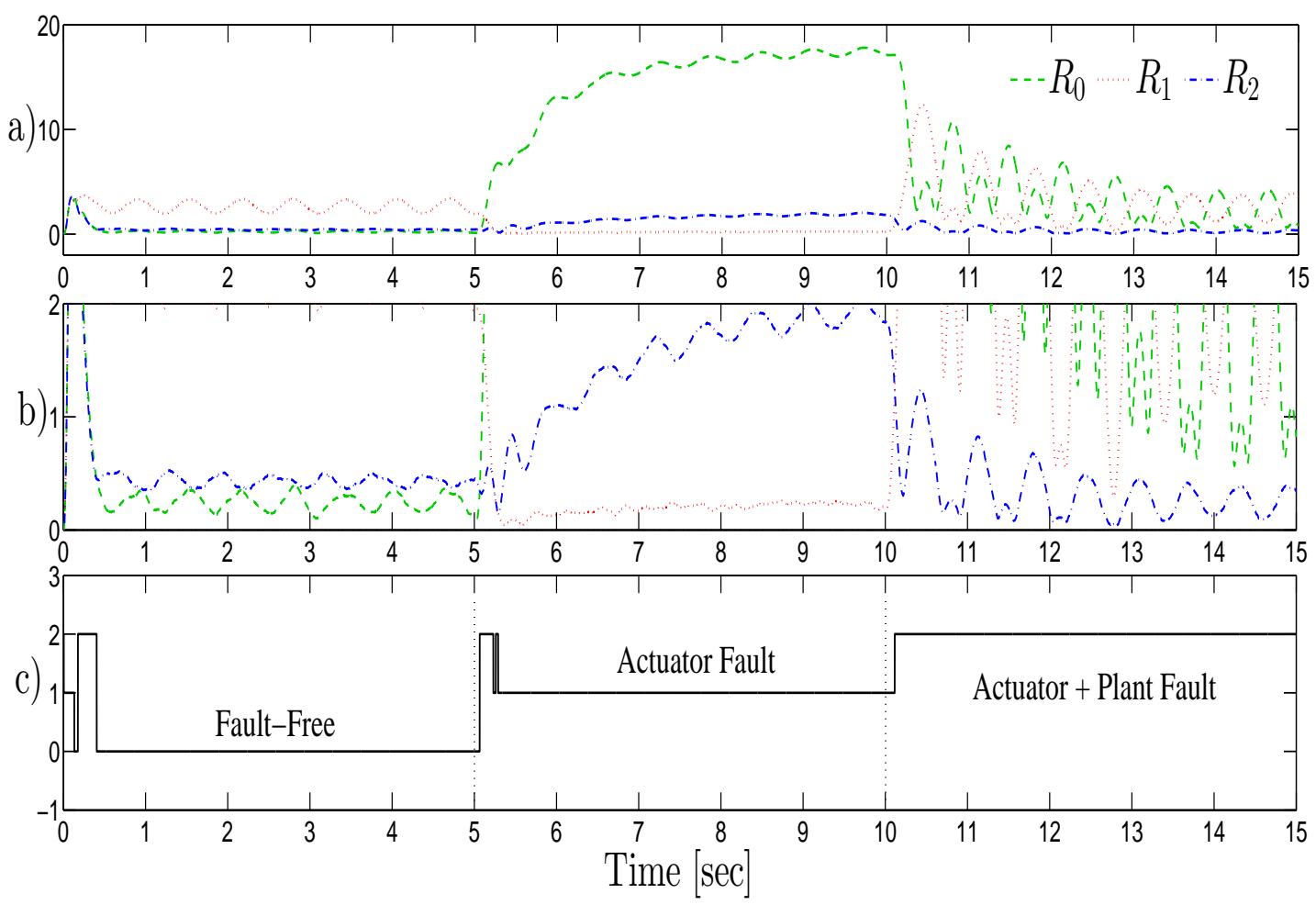

Figure 6. Residuals and Fault Detection Rule with Noise and Uncertainties. a) Residuals $R_{0}, R_{1}$ and $R_{2}$, $\tau_{\lambda}=0.06$. b) A zoom in a zero neighborhood of the residuals, $\tau_{\lambda}=0.06$. c) Fault detection rule, $\tau_{\lambda}=0.06$.

2. Chen J, Patton R. Robust model-based fault diagnosis for dynamic systems. Kluwer Acedmic Publishers: Boston, MA, 1999.

3. Blanke M, Kinnaert M, Lunze J, Staroswiecki M. Diagnosis and fault tolerant control. Springer: New York, 2003.

4. Zolghadri A. Advanced model-based fdir techniques for aerospace systems: Today challenges and opportunities. Progress in Aerospace Sciences 2012; 53:18-29.

5. Frank P, Ding X. Survey of robust residual generation and evaluation methods in observer-based fault detection systems. International Process Control 1997; 7(6):403-424.

6. Zolghadri A, Castang F, Henry D. Design of robust fault detection filters for multivariable feedback systems. International Journal of Modeling and Simulation 2006; 26:17-26.

7. Hammouri H, Kabore P, Kinnaert M. A geometric approach to fault detection and isolation for bilinear systems. IEEE Transactions on Automatic Control 2000; 46(9):1451-1455.

8. De-Persis C, Isidori A. A geometric approach to nonlinear fault detection and isolation. IEEE Transactions on Automatic Control 2001; 46(6):853-865.

9. Zhang Q, Basseville M, Benveniste A. Fault detection and isolation in nonlinear dynamic systems: A combined inputoutput and local approach. Automatica 1998; 34(11):1359-1373.

10. Efimov D, Zolghadri A, Raïssi T. Actuator fault detection and compensation under feedback control. Automatica 2011; 47(8):1699-1705.

11. Zolghadri A. A redundancy-based strategy for safety management in a modern civil aircraft. Control Engineering Practice 2000; 8(5):545-554.

12. Zhang X, Polycarpou M, Parisini T. A robust detection and isolation scheme for abrupt and incipient faults in nonlinear systems. IEEE Transactions on Automatic Control 2002; 47(4):576-593.

13. Xu A, Zhang Q. Nonlinear system fault diagnosis based on adaptive estimation. Automatica 2004; 40(7):11811193.

14. Efimov D, Cieslak J, Zolghadri A, Henry D. Actuator fault detection in aircraft systems: Oscillatory failure case study. Annual Reviews in Control 2013; DOI: 10.1016/j.arcontrol.2013.04.007.

15. Casavola A, Famularo D, Franze G. Robust fault detection of uncertain linear systems via quasi-lmis. Automatica 2008; 44:289-295. 
16. Efimov D, Cieslak J, Henry D. Supervisory fault-tolerant control with mutual performance optimization. International Journal of Adaptive Control and Signal Processing 2013; 27(4):251-279.

17. Edwards C, Spurgeon S, Patton R. Sliding mode observers for fault detection and isolation. Automatica 2000; 36(4):541-553.

18. Floquet T, Barbot J. An observability form for linear systems with unknown inputs. International Journal of Control 2006; 79:132-139.

19. Yan XG, Edwards C. Nonlinear robust fault reconstruction and estimation using sliding mode observer. Automatica September 2007; 43(9):1605-1614.

20. Fridman L, Levant A, Davila J. Observation of linear systems with unknown inputs via high-order sliding-modes. International Journal of System Science 2007; 38(10):773-791.

21. Alwi H, Edwards C, Tan C. Sliding mode estimation schemes for incipient sensor faults. Automatica 2009; 45:1679-1865.

22. Tan C, Edwards C. Robust fault reconstruction in uncertain linear systems using multiple sliding mode observers in cascade. IEEE Transactions on Automatic Control April 2010; 55(4):855-867.

23. Efimov D, Fridman L, Raïssi T, Zolghadri A, Seydou R. Application of interval observers and hosm differentiators for fault detection 2012; :516-521.

24. Rolink M, Boukhobza T, Sauter D. High order sliding mode observer for fault actuator estimation and its application to the three tanks benchmark. Workshop on Advanced Control and Diagnosis, 2006.

25. Levant A. High-order sliding modes: differentiation and output-feedback control. International Journal of Control 2003; 76(9-10):924-941.

26. Iqbal M, Bhatti AI, Iqbal S, Khan Q, Kazmi IH. Fault diagnosis of nonlinear systems using higher order sliding mode technique. Proceedings of the 7th Asian Control Conference, 2009; 875-880.

27. Maybeck PS. Multiple model adaptive algorithms for detecting and compensating sensor and actuator/surface failures in aircraft flight control systems. International Journal of Robust and Nonlinear Control 1999; 9(14):10511070.

28. Uppal FJ, Patton RJ. Neuro-fuzzy uncertainty de-coupling: a multiple-model paradigm for fault detection and isolation. International Journal of Adaptive Control and Signal Processing 2004; 19(4):281-304.

29. Rodrigues M, Theilliol D, Adam-Medina M, Sauter D. A fault detection and isolation scheme for industrial systems based on multiple operating models. Control Engineering Practice 2008; 16(2):225-239.

30. Menke TE, Maybeck PS. Sensor/actuator failure detection in the vista $\mathrm{f}-16$ by multiple model adaptive estimation. IEEE Transactions on Aerospace and Electronic Systems 1995; 31(4):1218-1229.

31. Boskovic JD, Mehra RK. Multiple-model adaptive flight control scheme for accommodation of actuator failures. Journal of Guidance, Control, and Dynamics 2002; 25(4):712-724.

32. White NA, Maybeck PS, DeVilbiss SL. Detection of interference/jamming and spoofing in a dgps-aided inertial system. IEEE Transactions on Aerospace and Electronic Systems 1998; 34(4):1208-1217.

33. Isidori A. Nonlinear Control Systems. Springer-Verlag: London, England, 1996.

34. Angulo M, Fridman L, Levant A. Output-feedback finite-time stabilization of disturbed lti systems. Automatica 2012; 48(4):606-611.

35. Filippov A. Differential Equations with Discontinuos Right-hand Sides. Kluwer Academic Publishers: Dordrecht, The Netherlands, 1988.

36. Khalil H. Nonlinear Systems. Prentice Hall: New Jersey, U.S.A., 2002.

37. Dávila A, Moreno JA, Fridman L. Optimal lyapunov function selection for reaching time estimation of super twisting algorithm. Proc. 48th IEEE Conf. Decision Control and 28th Chinise Control Conf., Shanghai, P.R. China, 2008; $8405-2410$.

38. Cruz-Zavala E, Moreno J, Fridman L. Uniform robust exact differentiator. IEEE Transactions on Automatic Control June 2011; 56(11):2727-2733.

39. Levant A. Robust exact differentiation via sliding mode technique. Automatica 1998; 34(3):379-384.

40. Alwi H, Edwards C, Tan C. Fault Detection and Fault-tolerant Control Using Sliding Modesl. AIC Seriese, SpringVerlag, 2011.

41. Davila J, Fridman L, Poznyak A. Observation and identification of mechanical systems via second order sliding modes. International Journal of Control 2006; 79(10):1251-1262.

42. Polyakov A, Fridman L. Stability notions and lyapunov functions for sliding mode control systems. Journal of the Franklin Institute; Http://dx.doi.org/10.1016/j.jfranklin.2014.01.002.

43. Utkin V, Guldner J, Shi J. Sliding Modes in Electromechanical Systems. Taylor and Francis: London, 1999. 